\title{
Self-regulation of inflammatory cell trafficking in mice by the leukocyte surface apyrase CD39
}

\author{
Matthew C. Hyman, ${ }^{1}$ Danica Petrovic-Djergovic, ${ }^{2}$ Scott H. Visovatti, ${ }^{2}$ Hui Liao, ${ }^{2}$ \\ Sunitha Yanamadala, ${ }^{2}$ Diane Bouïs, ${ }^{2}$ Enming J. Su, ${ }^{2}$ Daniel A. Lawrence, ${ }^{2}$ \\ M. Johan Broekman, ${ }^{3,4}$ Aaron J. Marcus, ${ }^{3,4,5}$ and David J. Pinsky',2
}

\begin{abstract}
1Department of Molecular and Integrative Physiology and 2Division of Cardiovascular Medicine, Department of Internal Medicine, University of Michigan Medical Center, Ann Arbor, Michigan, USA. ${ }^{3}$ Division of Hematology and Medical Oncology, Department of Medicine, Weill Cornell Medical College, New York, New York, USA. “Division of Hematology and Medical Oncology, Medical and Research Services, Veterans Affairs New York Harbor Healthcare System, New York, New York, USA. ${ }^{5}$ Department of Pathology and Laboratory Medicine, Weill Cornell Medical College, New York, New York, USA.
\end{abstract}

\begin{abstract}
Leukocyte and platelet accumulation at sites of cerebral ischemia exacerbate cerebral damage. The ectoenzyme CD39 on the plasmalemma of endothelial cells metabolizes ADP to suppress platelet accumulation in the ischemic brain. However, the role of leukocyte surface CD39 in regulating monocyte and neutrophil trafficking in this setting is not known. Here we have demonstrated in mice what we believe to be a novel mechanism by which CD39 on monocytes and neutrophils regulates their own sequestration into ischemic cerebral tissue, by catabolizing nucleotides released by injured cells, thereby inhibiting their chemotaxis, adhesion, and transmigration. Bone marrow reconstitution and provision of an apyrase, an enzyme that hydrolyzes nucleoside tri- and diphosphates, each normalized ischemic leukosequestration and cerebral infarction in CD39-deficient mice. Leukocytes purified from $\mathrm{Cd}_{39} 9^{-/-}$mice had a markedly diminished capacity to phosphohydrolyze adenine nucleotides and regulate platelet reactivity, suggesting that leukocyte ectoapyrases modulate the ambient vascular nucleotide milieu. Dissipation of $A T P$ by $C D 39$ reduced $P 2 X_{7}$ receptor stimulation and thereby suppressed baseline leukocyte $\alpha_{M} \beta_{2}$-integrin expression. As $\alpha_{M} \beta_{2}$-integrin blockade reversed the postischemic, inflammatory phenotype of $\mathrm{Cd39} 9^{-/}$mice, these data suggest that phosphohydrolytic activity on the leukocyte surface suppresses cell-cell interactions that would otherwise promote thrombosis or inflammation. These studies indicate that CD39 on both endothelial cells and leukocytes reduces inflammatory cell trafficking and platelet reactivity, with a consequent reduction in tissue injury following cerebral ischemic challenge.
\end{abstract}

\section{Introduction}

The early consequences of cerebral ischemia are often amplified by local leukocyte and platelet accumulation. This cellular accrual exacerbates cerebral damage by impeding distal microvascular flow $(1,2)$ and promoting local inflammation, tissue destruction, and regional edema. Although purinergic signaling is a critical mediator of platelet and leukocyte accumulation, endogenous mechanisms regulating these events are poorly understood (3-6). We have shown previously that platelet accumulation in the ischemic brain is suppressed by the endothelial ectonucleoside triphosphate diphosphohydrolase 1 (CD39), as well as by a soluble engineered fragment $(7,8)$, which catalyzes the terminal phosphohydrolysis of $\operatorname{ADP}$ and $\operatorname{ATP}(9,10)$. However, platelet reactivity and accumulation cannot fully account for the sequelae of cerebral ischemia (2). Though CD39 regulates platelet activation and adhesion through catabolism of $\operatorname{ADP}(7,11,12)$, it is not known whether $\operatorname{CD} 39$ modulates trafficking of inflammatory cells in ischemic tissue. Emerging evidence has highlighted a crucial role for autocrine and

Conflict of interest: The authors have declared that no conflict of interest exists. Nonstandard abbreviations used: APCP, adenosine $5^{\prime}$-( $\alpha, \beta$-methylene)diphosphate; ATP, adenosine 5 -triphosphate; BMDC, bone marrow-derived cell; bzATP, $2^{\prime}\left(3^{\prime}\right)$-O-(4-benzoylbenzoyl)ATP; MCA, middle cerebral artery; NGF, nerve growth factor; ox-ATP, periodate oxidized ATP; PI, propidium iodide; solCD39, a soluble form of human CD39.

Citation for this article: J. Clin. Invest. 119:1136-1149 (2009). doi:10.1172/JCI36433. paracrine purinergic signaling in the activation and chemotaxis of leukocytes (13). This led us to hypothesize that ectonucleotidases, which can dissipate adenine nucleotides and generate adenosine, may regulate inflammation in ischemic tissues.

Extracellular ATP and ADP, biomarkers of cellular injury (14), amplify inflammatory cascades by attracting additional leukocytes to sites of tissue damage. Thus, purine-mediated leukocyte trafficking, like ATP-directed neutrophil activation and chemotaxis, may exacerbate collateral tissue destruction following hypoxiainduced neuronal cell death $(13,15,16)$. Adenine nucleotides, however, are unique in that an endogenous and highly specific mechanism exists by which these signaling moieties could be degraded. Quenching of purinergic signaling would be a novel means to temper inflammation that would not require classical chemokine and cytokine signals. Furthermore, AMP, generated by CD39-mediated phosphohydrolysis of ATP/ADP, is subsequently converted to adenosine (17-19). In this schema, nucleotide metabolism and nucleoside generation mediate a critical balance between inflammatory and quiescent signals, with vascular apyrases such as CD39 mediating the fulcrum/pivot point between the two.

In the present study, we tested the hypothesis that CD39 regulates leukocyte trafficking by rapidly dissipating ATP and ADP from the leukocyte microenvironment in the ischemic brain. Experiments were designed to determine whether CD39 alters leukocyte accumulation in the ischemic brain and, if so, whether the mecha- 
A
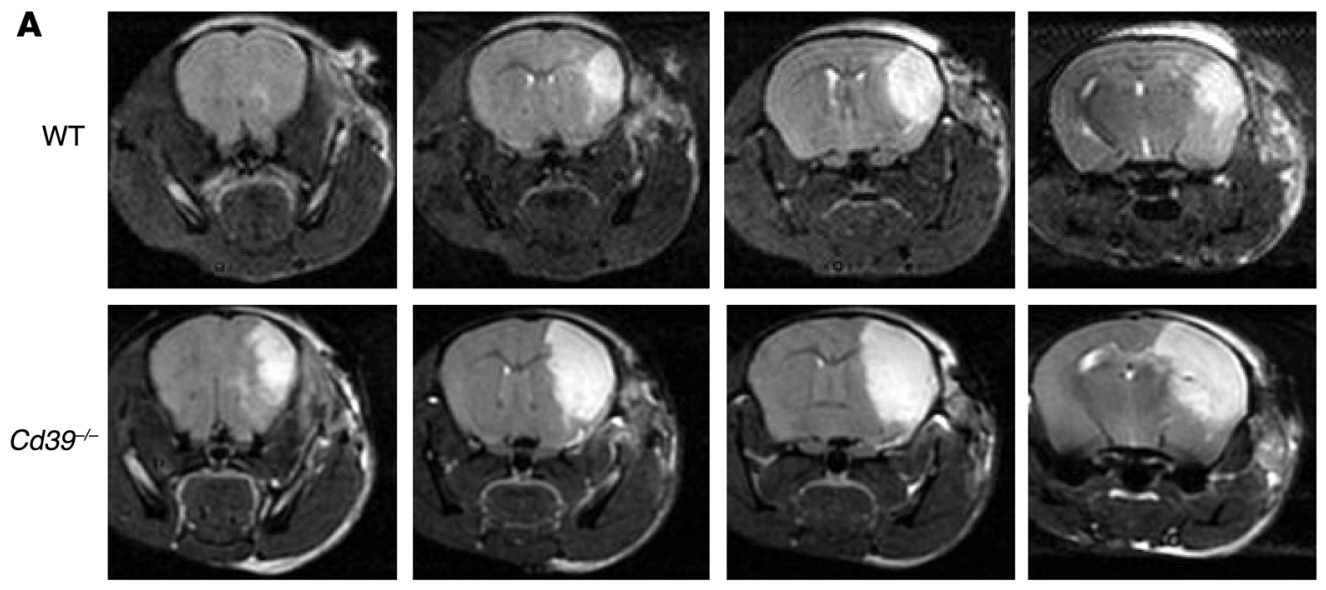

B

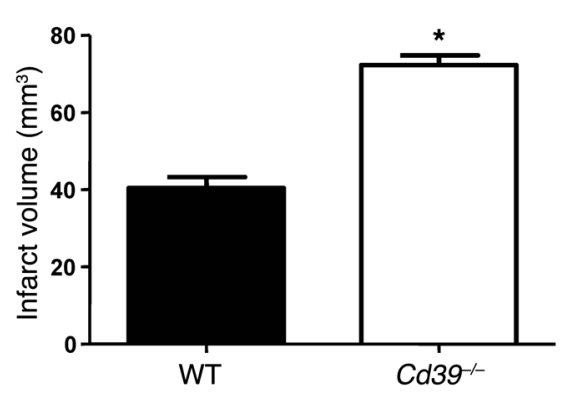

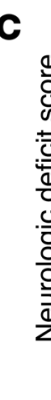

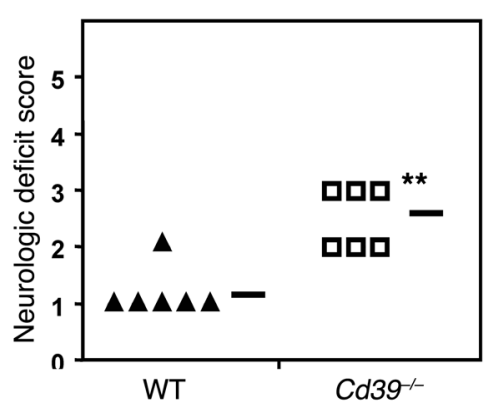

Figure 1

Effect of Cd39 genotype on resistance to cerebral ischemia 48 hours after MCA occlusion. (A) Representative magnetic resonance images of WT and Cd39-/- brains after cerebral ischemia. (B) Quantification of average cerebral infarct volume in ischemic WT and Cd39-/brains. (C) Functional effects of cerebral infarction were assessed using a neurologic deficit score, with higher scores indicating a greater deficit (50). The horizontal bars indicate the average neurologic deficit score for each group. $n=6$ per group; ${ }^{\star} P<0.03,{ }^{\star \star} P<0.01$. nism is dependent upon endothelial or leukocyte CD39 expression or both. Previous studies focused primarily on endothelial CD39 and thrombosis; however, the role of CD39 on the leukocyte cell surface in ischemic tissue is unknown. Employing an experimental strategy of bone marrow transplantation and selective reconstitution, we now demonstrate CD39-dependent leukocyte trafficking into an ischemic tissue and elucidate the relative contributions of CD39-bearing cell subpopulations to ischemic leukosequestration. Furthermore, the $\mathrm{P} 2 \mathrm{X}_{7}$ receptor is identified as a regulator of $\alpha_{M} \beta_{2}$-integrin expression, with CD39 downregulating $\alpha_{M} \beta_{2}$-integrin by suppressing stimulation of leukocyte $\mathrm{P} 2 \mathrm{X}_{7}$. These studies show that leukocytes, through the action of CD39, regulate their own recruitment to postischemic tissue.

\section{Results}

Cd39 genotype and clinical sequelae of cerebral ischemia. Permanent cerebral ischemia was induced in CD39-deficient and WT mice with the use of a photothrombotic model of middle cerebral artery (MCA) occlusion (20). Forty-eight hours later, T2-weighted cortical MRIs were performed to assess infarct volume (Figure 1A). Infarct volumes in CD39-deficient mice were $78.7 \%$ larger than those in WT controls (Figure 1B). The larger infarct volumes in $\mathrm{Cd} 39^{-/-}$mice were of functional significance, since they also demonstrated greater neurological deficits than WT mice (Figure 1C). The data obtained with this model of stroke parallel those previously reported with intraluminal MCA occlusion using a nylon monofilament - studies in which larger cerebral infarct volumes were observed in $\mathrm{Cd} 39^{-/-}$mice 24 hours after ischemia (7).

CD39 modulation of ischemic leukosequestration. CD39-deficient mice are characterized by enhanced platelet deposition in ischemic cerebral tissue, yet platelet activation alone cannot account for all of the sequelae of cerebral infarction. For instance, when a short-acting
GPIIb/IIIa antagonist was given to mice in the setting of stroke, although platelet deposition was markedly diminished, there was reduction in, but not complete rescue from, cerebral infarction (2). We hypothesized that the ischemia susceptibility of $\mathrm{Cd} 39^{-/-}$mice could be attributed not only to release by platelets of granular contents, exposure of platelet surface adhesion molecules such as P-selectin, and provision of a procoagulant phospholipid surface, but also to increased postischemic leukocyte infiltration. Histologic examination revealed that large numbers of macrophages and neutrophils are recruited to ischemic cortex and suggested that absence of CD39 in particular exaggerates macrophage recruitment into the ischemic zone (Figure 2, A-L). Using flow cytometry to quantify leukocyte infiltration, we analyzed ischemic and nonischemic hemispheres of mice 48 hours after ischemia induction. Antibodies against CD45 and LY-6G characterized the neutrophil (CD45 hi LY- $6 \mathrm{G}^{+}$) subpopulation of the infiltrating cells (green in Figure $2 \mathrm{M})$; F4/80 positivity in combination with CD45 staining identified macrophages $\left(\mathrm{CD} 45^{\mathrm{hi}} \mathrm{F} 4 / 80^{+}\right.$) (blue in Figure $\left.2 \mathrm{~N}\right)(21,22)$. The $\mathrm{CD} 45^{\text {lo }} \mathrm{F} 4 / 80^{+}$cells observed could represent activated microglial cells, which are known to express F4/80 (23, 24). Ischemic hemispheres of untransplanted CD39-deficient mice demonstrated $61 \%$ more total infiltrating nucleated cells as compared with the ischemic hemispheres of untransplanted WT mice. Conversely, in nonischemic hemispheres of controls, the numbers of infiltrating cells did not differ significantly between genotypes (data not shown). This implied that CD39 does not affect baseline numbers of resident leukocytes in the brain. Subpopulation analysis demonstrated a 2-fold enrichment in the number of infiltrating neutrophils and macrophages in each ischemic hemisphere of CD39-deficient versus control mice $\left(259 \times 10^{3} \pm 24 \times 10^{3}\right.$ vs. $134 \times 10^{3} \pm 4 \times 10^{3}$ neutrophils per hemisphere and $108 \times 10^{3} \pm 5 \times 10^{3}$ vs. $46 \times 10^{3} \pm$ $3 \times 10^{3}$ macrophages per hemisphere) (Figure $2, \mathrm{O}$ and $\mathrm{P}$ ). 

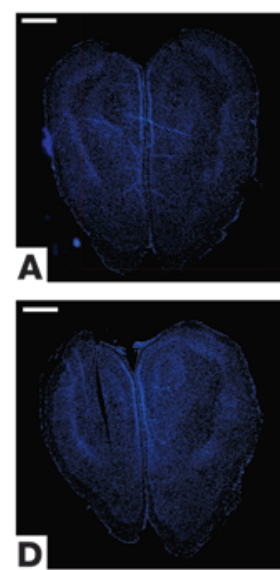

$B$
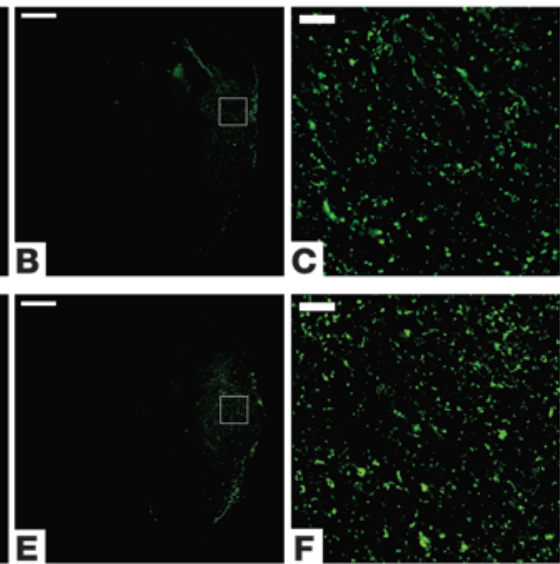

M

WT isotype

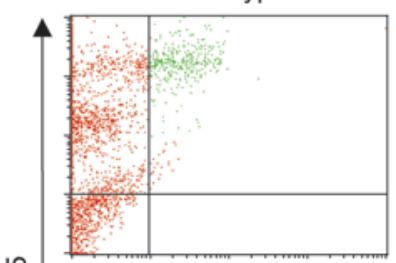

号

Cd39-- isotype
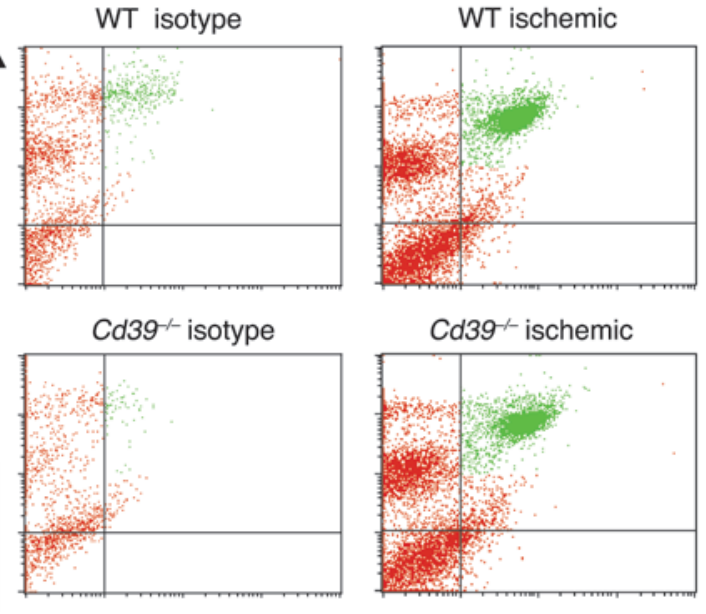

LY-6G

N
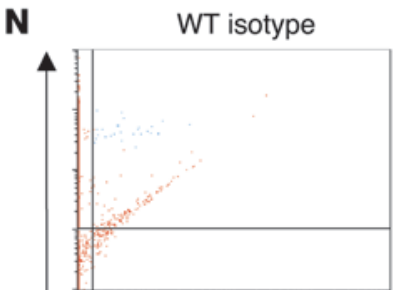

ปั

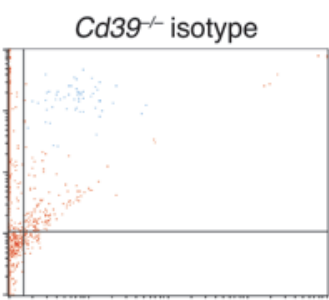

WT ischemic

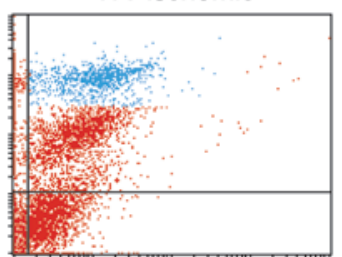

Cd $39^{-/}$ischemic

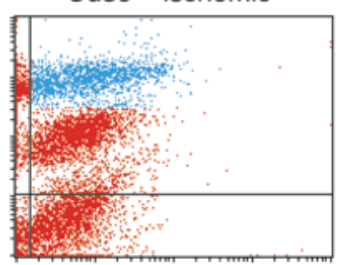

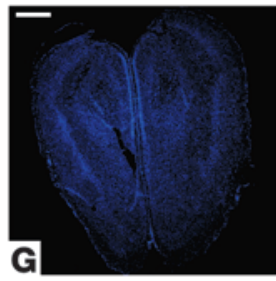

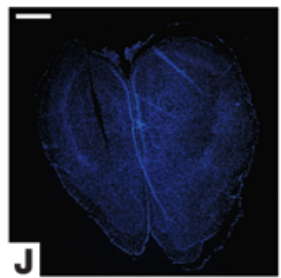

WT contralateral

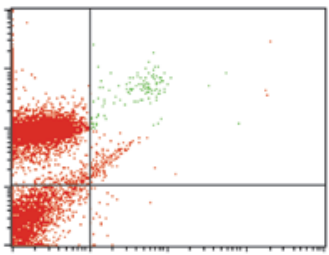

Cd $39^{-}$contralateral

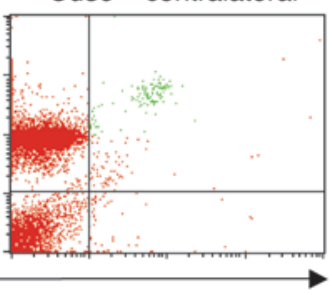

WT contralateral

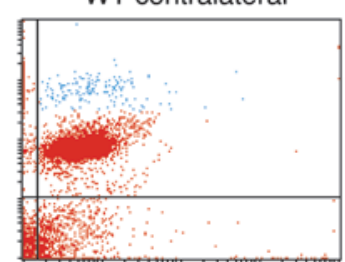

Cd $39^{--}$contralateral

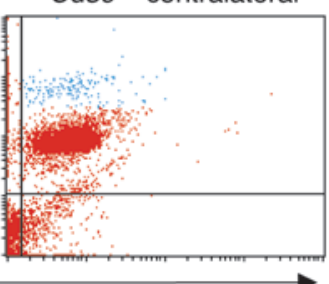

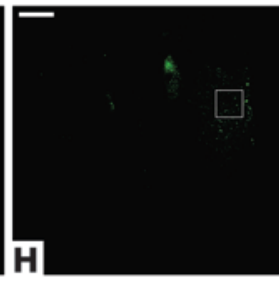
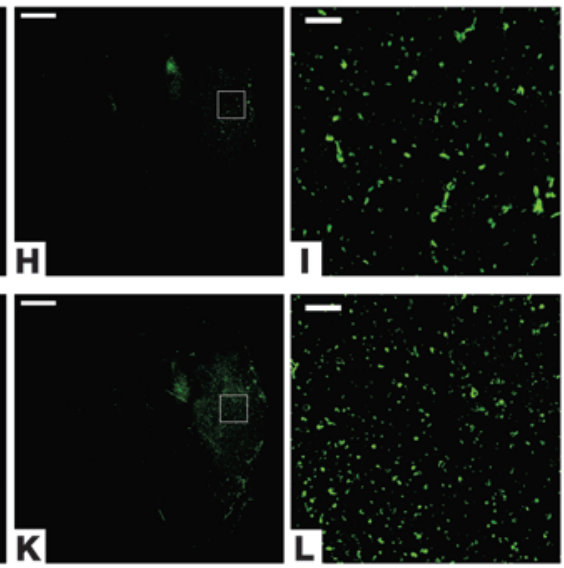

O

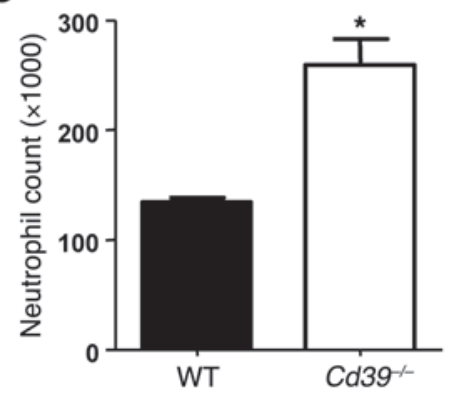

$\mathbf{P}$

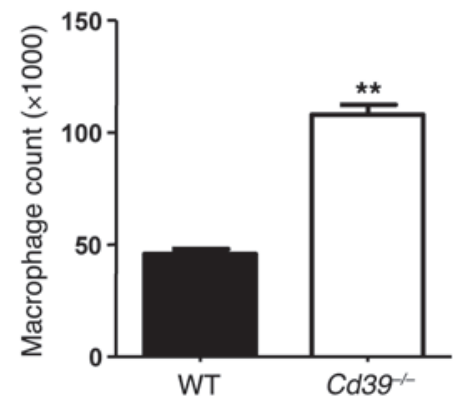

Figure 2

$\mathrm{F} 4 / 80$

Role of CD39 in leukocyte sequestration in the ischemic cerebrum. The ischemic brains of WT (A-C) and Cd39-/- (D-F) mice were stained for nuclei (A and D) and neutrophils (B, C, E, and F). Scale bars: 1,000 $\mu \mathrm{m}(\mathbf{A}, \mathbf{B}, \mathbf{D}$, and E), $100 \mu \mathrm{m}(\mathbf{C}$ and $\mathbf{F})$. Adjacent sections of WT (G-I) and

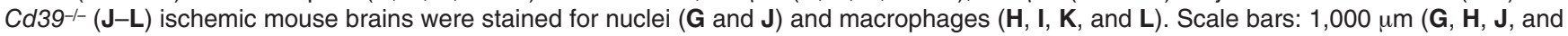
$\mathbf{K}), 100 \mu \mathrm{m}$ (I and $\mathbf{L})$. The white boxes in the center panels show the magnified area in the right panels. (M) Representative scattergrams of LY-6G-stained neutrophil populations (green) within the ischemic and contralateral hemispheres of WT and Cd39-/- mice as well as isotype control. (N) Representative scattergrams of F4/80-stained macrophage populations (blue) within the ischemic and contralateral hemispheres of WT and Cd39-/- mice as well as isotype control. The effect of CD39 on the infiltration of leukocyte subpopulations was assessed using flow cytometry: neutrophils $(\mathbf{O})$ and macrophages $(\mathbf{P}) . n=6$ per group in $\mathbf{M}-\mathbf{P} ;{ }^{*} P<0.005$, ${ }^{\star *} P<0.0001$. 
A

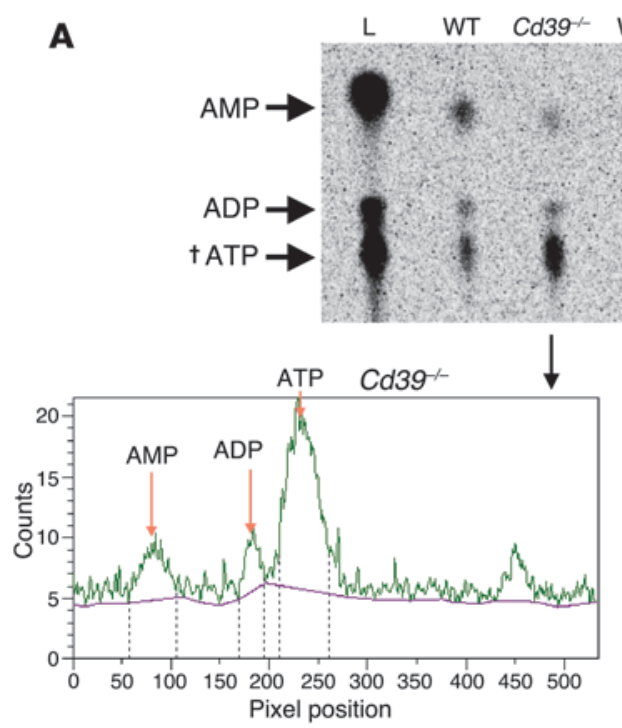

WT Cd39 ${ }^{\circ}$ WT Cd39

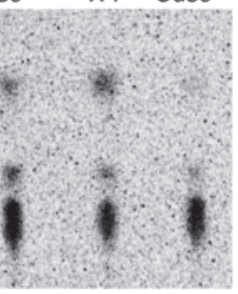

WT

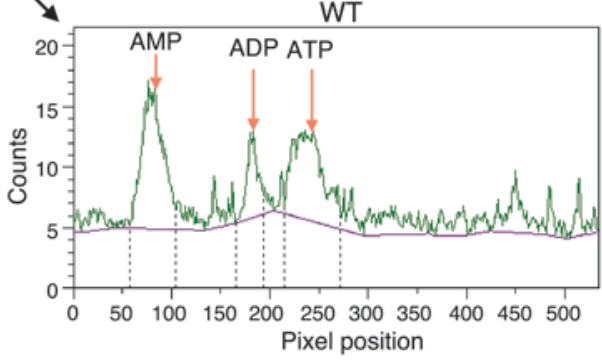

B

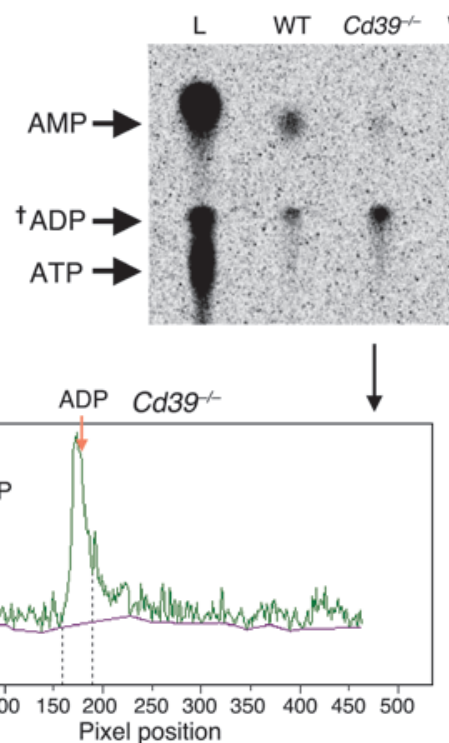

Pixel position

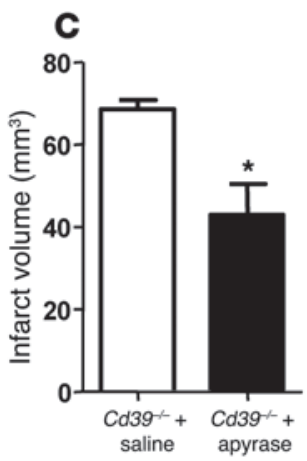

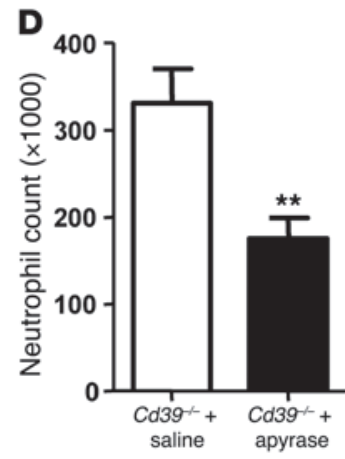

WT $\mathrm{Cd}_{39^{\circ}}$ WT $\mathrm{Cd} 39^{-}$
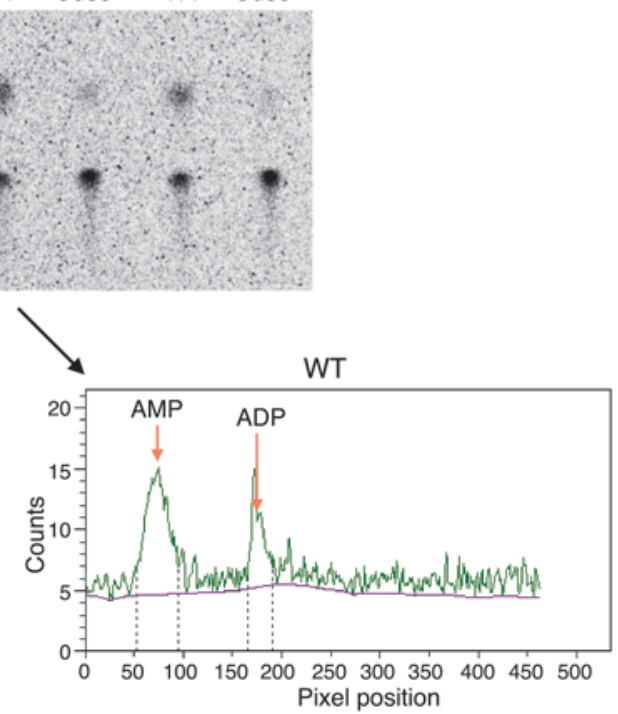

E

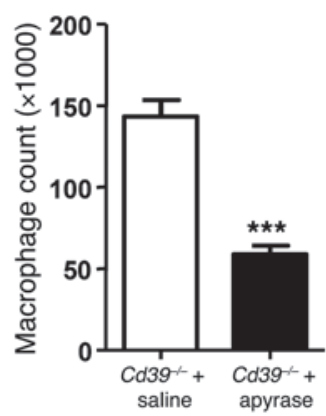

\section{Figure 3}

Circulating ectoapyrase activity confers resistance to cerebral ischemia. Leukocytes purified from WT and Cd39-- mice were coincubated with $\left[8-{ }^{-14} \mathrm{C}\right] \mathrm{ATP}$ or $\left[\mathrm{8}^{-14} \mathrm{C}\right] \mathrm{ADP}$ to assess the functional importance of leukocyte CD39. Metabolic products were resolved via TLC, and representative radioactivity histograms are shown for ATP (A) and ADP (B) (L, ladder comprising radiolabeled ATP, ADP, and AMP). Forty-eight hours after ischemia induction, MRI was performed to assess the therapeutic potential of soluble apyrase in diminishing cerebral infarction. Crosses $(\dagger)$ indicate the metabolite added to the reaction $(\mathbf{C})$ Multiple strokes were scored to generate aggregate cerebral infarct volumes. Additional mice were subjected to flow cytometric analysis to determine neutrophil (D) and macrophage (E) infiltration following apyrase administration. $n=4$ per group in $\mathrm{C}-\mathrm{E}$; ${ }^{*} P<0.04$, ${ }^{\star *} P<0.02,{ }^{* *} P<0.001$.
Circulating apyrase protects $\mathrm{Cd} 39^{-/-}$mice from cerebral ischemia. We hypothesized that the heightened leukocyte flux seen in $\mathrm{Cd} 39^{-/-}$mice was due in part to an absence of vascular wall CD39 activity, and also in part due to a loss of CD39 circulating on the leukocytes themselves. To examine this, we assessed the ability of WT and CD39-deficient leukocytes to metabolize ATP and ADP in their extracellular milieu. Purified buffy coats were incubated with either $\left[8-{ }^{14} \mathrm{C}\right] \mathrm{ADP}$ or $\left[8-{ }^{14} \mathrm{C}\right]$ ATP, after which phosphohydrolysis was assessed by TLC (Figure 3, A and B). CD39-deficient leukocytes were deficient in ATPase and ADPase activity (Figure $3, \mathrm{~A}$ and $\mathrm{B}$ ). To demonstrate that the loss of ectoapyrase activity in Cd39-null mice was driving their leukosequestration phenotype, a soluble apyrase was administered prior to ischemic induction. Apyrase is a functional analog of CD39 that has been shown to restore normal vascular homeostasis (mitigate platelet 

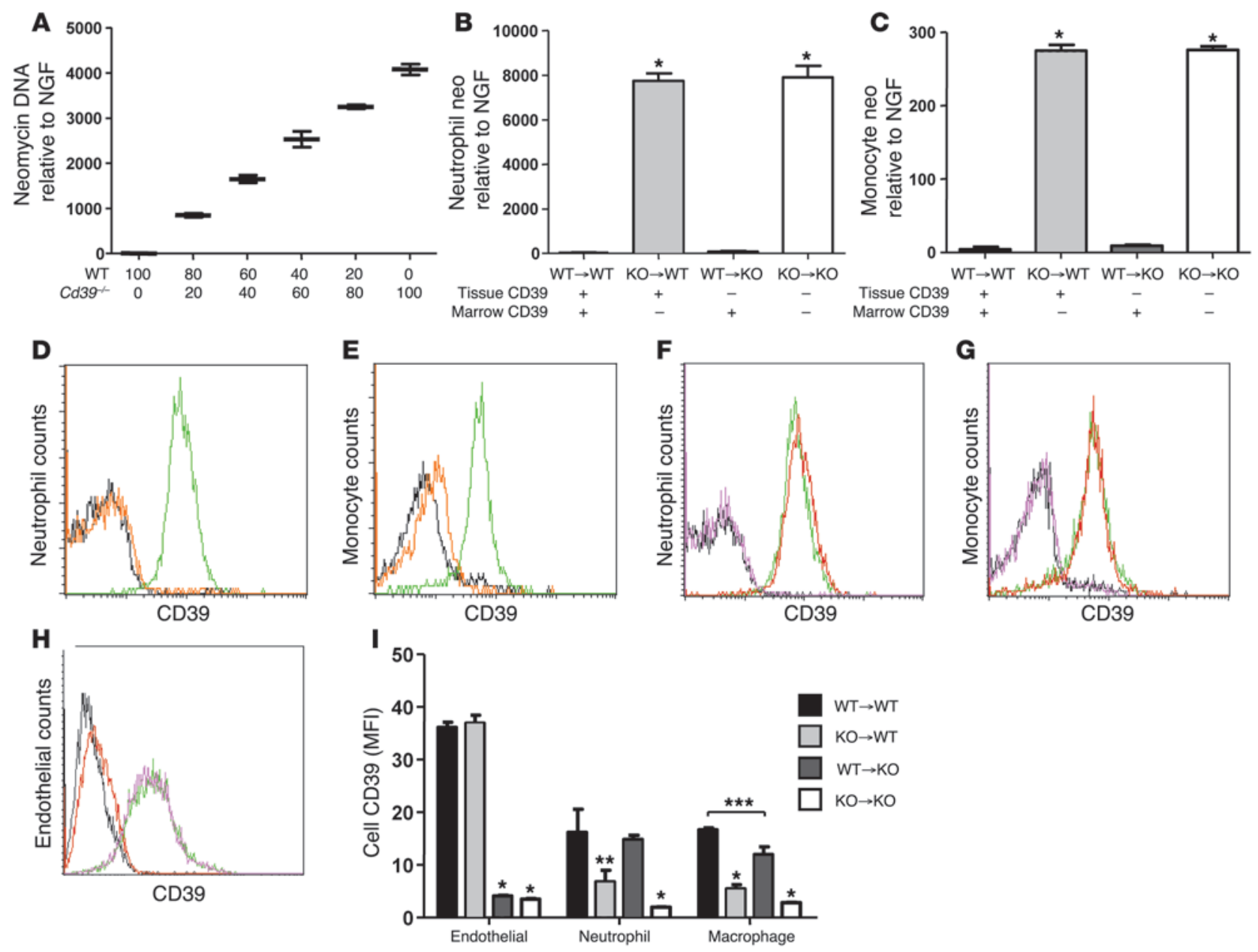

Figure 4

CD39 deficiency does not impair bone marrow reconstitution. To determine the contribution of donor and recipient cells to the neutrophil and monocyte subpopulations, we developed a quantitative PCR that measured neomycin gene dosage. (A) Mixtures of WT and Cd39-/- peripheral blood leukocytes ( $x$ axis indicates cell number $\times 10,000$ ) were used to validate this assay. Leukocyte buffy coats from bone marrow-transplanted mice were sorted by flow cytometry to isolate the neutrophil and monocyte subpopulations. The neutrophils (B) and monocytes (C) were then quantified for relative neomycin DNA. Peripheral blood from untransplanted WT (green) and Cd39--- (black) mice was separated into neutrophil (D) and monocyte (E) populations by flow cytometry and then examined for CD39 expression. Isotype control is shown in orange. Peripheral leukocytes from bone marrow chimeric mice were sorted into neutrophil $(\mathbf{F})$ and monocyte $(\mathbf{G})$ populations and stained for CD39: WT $\rightarrow$ WT (green), WT $\rightarrow \mathrm{KO}$ (red), KO $\rightarrow \mathrm{WT}$ (magenta), and $\mathrm{KO} \rightarrow \mathrm{KO}$ (black). Whole-lung homogenates from bone marrow chimeric mice were sorted for endothelial cells $(\mathbf{H})$ and stained for CD39: WT $\rightarrow$ WT (green), WT $\rightarrow \mathrm{KO}$ (red), KO $\rightarrow$ WT (magenta), and KO $\rightarrow \mathrm{KO}$ (black). (I) Whole-lung digests from bone marrow-transplanted mice were analyzed for relative CD39 expression on endothelial, neutrophil, and macrophage subpopulations. $n=4$ or 5 per group; ${ }^{*} P<0.001,{ }^{* \star} P<0.01,{ }^{* *} P<0.05$.

desensitization) in Cd39-/- mice (11). Cd39-null mice treated with apyrase, but not saline, were protected, with a diminished cerebral infarct size $\left(42.8 \pm 7.6 \mathrm{~mm}^{3}\right.$ vs. $68.6 \pm 2.1 \mathrm{~mm}^{3}, P<0.04$ (Figure $\left.3 \mathrm{C}\right)$. This finding is concordant with previous studies using solCD39, a soluble form of human CD39 (25) in murine (7) and rat (8) models of cerebral ischemia. Data also showed a concordant decrease in the number of neutrophils and macrophages in the ischemic brain of apyrase-treated mice when compared with saline controls (apyrase: $176 \times 10^{3} \pm$ $23 \times 10^{3}$ neutrophils per hemisphere vs. saline: $331 \times 10^{3} \pm 40 \times 10^{3}$ neutrophils per hemisphere, $P<0.02$; apyrase: $60 \times 10^{3} \pm 6 \times 10^{3}$ macrophages per hemisphere vs. saline: $144 \times 10^{3} \pm 10 \times 10^{3}$ macrophages per hemisphere, $P<0.001$ ) (Figure 3, D and E). These data show that inhibition of ischemia-driven leukocyte accumulation can be achieved through administration of a functional CD39 analog.
Restoration of normal leukocyte mobilization in $\mathrm{Cd} 39^{-1-}$ mice by bone marrow transplantation. To determine whether CD39 on circulating cells or on vascular tissue was contributing to the increased cerebral ischemia susceptibility of CD39-deficient animals, a series of bone marrow transplantations were performed between Cd39-null and WT animals (26). Cd39-/- or WT bone marrow was transplanted into myeloablated $\mathrm{Cd} 39^{---}(\mathrm{KO} \rightarrow \mathrm{KO})$ or $\mathrm{WT}(\mathrm{WT} \rightarrow \mathrm{WT})$ mice, respectively (donor $\rightarrow$ recipient). These mice served as transplantation controls. Similarly, $\mathrm{Cd} 39^{-/-}$or WT bone marrow was also transplanted into WT $(\mathrm{KO} \rightarrow \mathrm{WT})$ or $\mathrm{Cd} 39^{-1-}(\mathrm{WT} \rightarrow \mathrm{KO})$ myeloablated recipients to generate chimeric mice with $\mathrm{CD} 39$ either on the resident tissue alone $(\mathrm{KO} \rightarrow \mathrm{WT})$ or on bone marrow-derived cells (BMDCs) alone (WT $\rightarrow \mathrm{KO}$ ).

To confirm the efficiency of the marrow reconstitution, we developed a new quantitative PCR methodology with probe and primer 

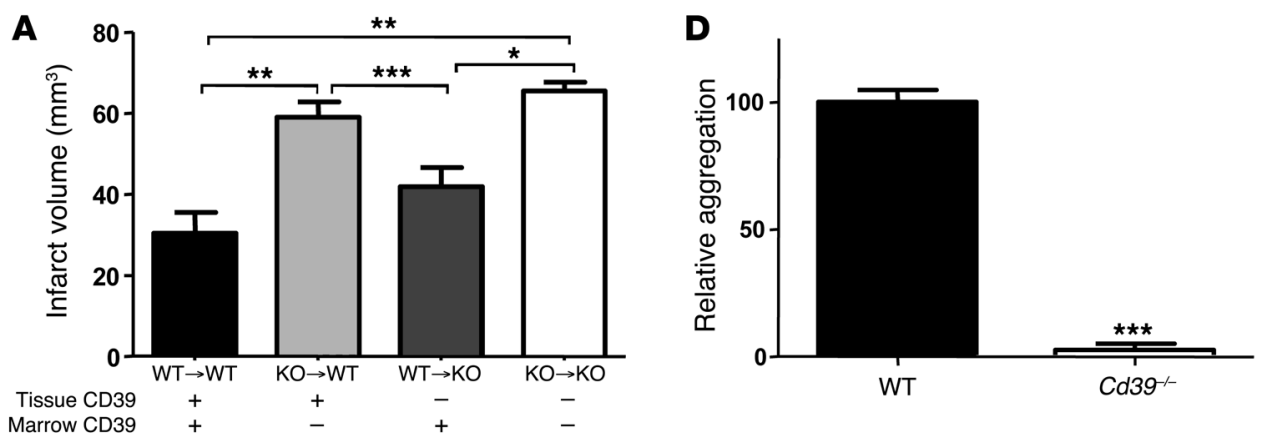

\section{Figure 5}

Role of CD39-bearing subpopulations in resistance to cerebral ischemia and regulation of platelet reactivity. WT and $\mathrm{Cd} 39^{-/-}$mice underwent bone marrow reconstitution to generate chimeric mice, as a means to explore selective ablation of CD39 in endothelial and leukocyte subpopulations. (A) Quantification of average cerebral infarct volume determined by MRI in ischemic chimera brains. The contribution of CD39 on endothelium and leukocytes to leukosequestration of neutrophils (B) and macrophages (C) was examined in chimeric mice. (D) Whole-blood platelet aggregometry with ADP stimulation was performed on WT and $\mathrm{Cd} 39^{-/-}$mice. Marrow-reconstituted mice were used to explore platelet reactivity following selective ablation of CD39 in endothelial and leukocyte subpopulations. (E) Representative whole-blood platelet aggregation profiles of each of the $4 \mathrm{chi}$ meric subpopulations 2 weeks after transplantation. (F) Quantification of average platelet aggregation profiles. $n=5$ or 6 per group in A-D; $n=4$ or 5 per group in $\mathbf{E}$ and $\mathbf{F}$. ${ }^{*} P<0.01$; ${ }^{* \star} P<0.001 ;{ }^{* \star *} P<0.005 ;{ }^{\dagger} P<0.01$ versus all other columns; $¥ P<0.02$; $\S P<0.001$ versus all other groups.

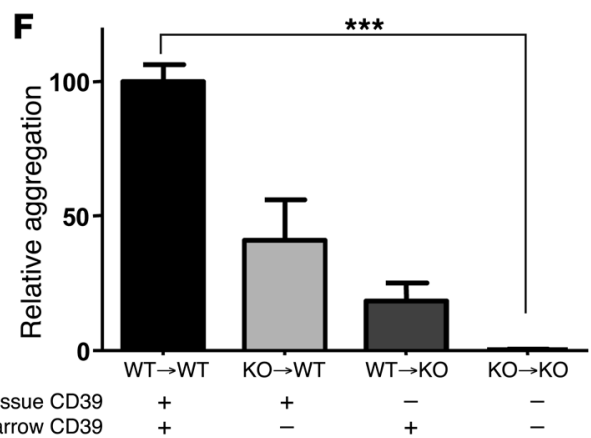

sets designed against both neomycin (cassette used in generating the Cd39 knockout) and nerve growth factor (NGF; as an internal control). This allowed assay of the percentage of cells in a population that were CD39-deficient (containing neomycin) or WT (no neomycin) (Figure 4A). Fluorescence-activated cell sorting was used to collect individual neutrophil and monocyte populations for DNA isolation. The Neo/ NGF assay was used to genotype the cells in each of our 4 chimeras and revealed that circulating neutrophils and monocytes were fully reconstituted (Figure 4, B and C). In a separate set of experiments, flow cytometry (Figure 4, D-G) was used to examine both the circulating neutrophil and monocyte protein expression of CD39. These methods demonstrated that at both the DNA and protein levels, neutrophils and monocytes were completely reconstituted in the chimeric mice. Further staining of tissue homogenates demonstrated that the resident endothelial populations retained the recipient phenotype following bone marrow transplantation (Figure 4I), with endothelial cells having approximately twice the surface CD39 expression of either the resident neutrophil or macrophage populations.

Eight to ten weeks were allowed for full bone marrow reconstitution, after which the chimeras were subjected to photothrombotic MCA occlusion. MRIs of the homologously transplanted (i.e., WT $\rightarrow$ WT or $\mathrm{KO} \rightarrow \mathrm{KO}$ ) and infarcted mice demonstrated that bone marrow transplantation does not alter the susceptibility to cerebral injury in ischemic stroke of WT and $\mathrm{Cd} 39^{-1-}$ mice. Mice without CD39 $(\mathrm{KO} \rightarrow \mathrm{KO})$ had significantly larger infarct volumes than mice with CD39 (WT $\rightarrow$ WT) $\left(65.6 \pm 2.3 \mathrm{~mm}^{3}\right.$ vs. $30.5 \pm 5.1 \mathrm{~mm}^{3}, P<0.001$ ) (Figure 5A). Chimeric mice with CD39bearing BMDCs were largely rescued from infarction when compared with $\mathrm{KO} \rightarrow \mathrm{KO}$ mice $\left(65.6 \pm 2.3 \mathrm{~mm}^{3}\right.$ vs. $42.2 \pm 4.7 \mathrm{~mm}^{3}$, $P<0.01)$. Conversely, expression of CD39 on only the vascular tissue surface provided limited protection from cerebral ischemia $\left(\mathrm{KO} \rightarrow \mathrm{WT}, 59.0 \pm 3.4 \mathrm{~mm}^{3}\right)$. Furthermore, the increased infarct volumes of the $\mathrm{KO} \rightarrow \mathrm{KO}$ mice proved to be functionally important, as they had significantly greater neurologic deficits than $\mathrm{WT} \rightarrow \mathrm{WT}$ or WT $\rightarrow$ KO mice (data not shown). KO $\rightarrow$ WT chimeras had an intermediate neurologic deficit.

Given the susceptibility of CD39-deficient mice to ischemiadriven leukosequestration, we examined which CD39-bearing tissues confer protection to WT mice using the same marrow reconstitution strategy. The total number of cells infiltrating the contralateral, nonischemic hemisphere was similar across all chimeric strains (data not shown). Subpopulation analysis of ischemic hemispheres showed that CD39 on BMDCs strongly suppressed leukocyte recruitment to ischemic tissue. The total number 
A

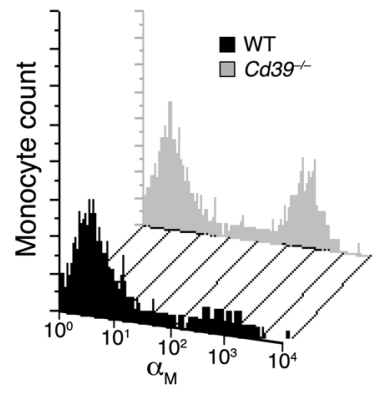

C

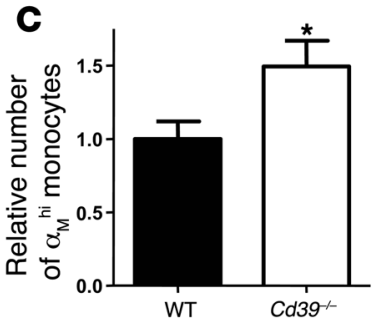

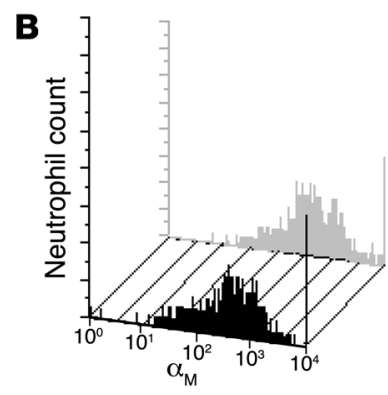

D

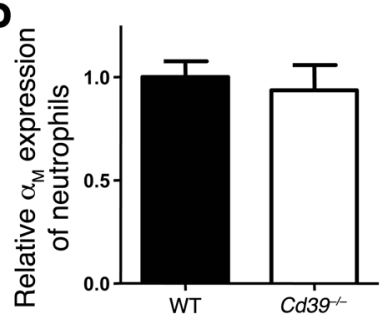

Figure 6

CD39 modulates circulating leukocyte $\alpha_{M} \beta_{2}$-integrin expression. Unstimulated whole blood of WT (black) and Cd39-/- (gray) mice was examined for $\alpha_{M} \beta_{2}$-integrin expression by staining the $\alpha_{M}$ subunit in the monocyte (A) and neutrophil (B) populations. The relative expression of $\alpha_{M}$ on the monocyte (C) and neutrophil (D) populations in WT and Cd $39^{-/-}$mice is shown. $n=9$ per group; ${ }^{\star} P<0.01$.

of infiltrating neutrophils was significantly higher $(P<0.01)$ in $\mathrm{KO} \rightarrow \mathrm{KO}$ mouse brain $\left(390 \times 10^{3} \pm 18 \times 10^{3}\right)$ compared with all other groups (WT $\rightarrow \mathrm{WT}, 217 \times 10^{3} \pm 16 \times 10^{3} ; \mathrm{KO} \rightarrow \mathrm{WT}$, $273 \times 10^{3} \pm 28.6 \times 10^{3} ; \mathrm{WT} \rightarrow \mathrm{KO}, 255 \times 10^{3} \pm 21 \times 10^{3}$ ) (Figure 5B). The number of macrophages recruited to the ischemic brain correlated closely with the presence or absence of CD39 on BMDCs. The absolute number of infiltrating macrophages was significantly increased, almost 2 -fold, in the $\mathrm{KO} \rightarrow \mathrm{KO}$ and $\mathrm{KO} \rightarrow \mathrm{WT}$ mouse brains when compared with either WT $\rightarrow$ WT or WT $\rightarrow$ KO mice (Figure 5C). This implies that although an acute rescue from CD39 deficiency can be obtained through administration of an apyrase or solCD39 analog, a permanent rescue can be obtained via bone marrow reconstitution with CD39-bearing cells.

CD39-bearing subpopulations contribute to platelet reactivity. CD39 has previously been shown to be a prime regulator of platelet activation and recruitment in vivo $(7,11,27)$ and ex vivo $(9,27)$, yet the role of CD39 loss from a subpopulation has not been explored. We hypothesized that $\mathrm{CD} 39$ on the surface of leukocytes could be regulating platelet activation and recruitment, thereby contributing to the sequelae of cerebral ischemia in this fashion. To determine how a change in leukocyte phosphohydrolytic activity might modulate platelet reactivity, we employed whole-blood aggregometry. In

\section{Figure 7}

Apyrase treatment modulates monocyte $\alpha_{M} \beta_{2}$-integrin expression. WT and $\mathrm{Cd} 39^{-/-}$mice were treated with soluble apyrase before examination of monocyte $\alpha_{M} \beta_{2}$-integrin expression. (A) Representative histogram shifts can be seen between vehicle-treated and apyrase-treated monocytes in WT and Cd39-/- mice. The aggregate effect of apyrase treatment on monocyte $\alpha_{M}$ expression can be seen in B. $n=4$ per group; ${ }^{*} P<0.01$ versus all other columns. contrast to platelet-rich plasma aggregometry, this assay mimics platelet-leukocyte interactions. In keeping with previous published observations in platelet-rich plasma aggregometry $(7,11)$, nontransplanted $\mathrm{Cd} 39^{-/-}$mice demonstrated platelet desensitization when compared with WT control mice (Figure 5D). We sought to examine whether this was attributable to the catabolism of platelet-activating nucleotides by endothelial cell- or leukocyte-bound CD39 using bone marrow reconstitution studies. Two weeks following reconstitution, mice with either tissue CD39 alone $(\mathrm{KO} \rightarrow \mathrm{WT})$ or BMDCs with CD39 (WT $\rightarrow$ KO) demonstrated only partial desensitization of platelets, demonstrating a role for both of these subpopulations in the maintenance of vascular homeostasis (Figure 5, E and F). This finding is complementary to previous work in which human CD39 was overexpressed on either the leukocyte or endothelial populations. These experiments showed that $\mathrm{CD} 39$ can regulate platelet activation from either the endothelial or leukocyte compartment (27).

CD39 deficiency and leukocyte $\alpha_{M} \beta_{2}$-integrin surface expression. ATP is known to upregulate expression of $\alpha_{M} \beta_{2}$-integrin (also known as MAC-1, CD11b/CD18), a critical glycoprotein adhesion receptor expressed on human neutrophils $(16,28)$. To discern whether a lack of CD39 resulted in stimulation of CD39-deficient leukocytes via basal nucleotide release, we examined peripheral blood monocyte and neutrophil populations by flow cytometry. Histologic analysis performed to confirm the cell gates revealed $92 \%$ and $97 \%$ purity of the sorted monocytes and neutrophils, respectively (Supplemental Figure 1; supplemental material available online with this article; doi:10.1172/JCI36433DS1). There was a 50\% increase in the number of $\alpha_{M} \beta_{2}$-integrin high-expressing monocytes (measured by the $\alpha_{M} /$ CD11b subunit) in Cd39-/- mice compared with WT controls (Figure 6, A and C). In contrast, neutrophils did not display differences in cell-surface $\alpha_{M} \beta_{2}$-integrin expression at baseline (Figure 6, B and D). Treatment of $C d 3^{-/-}$animals with a soluble apyrase restored expression of $\alpha_{M} \beta_{2}$-integrin on monocytes to near-WT levels (Figure 7). By comparison, treatment of WT animals with
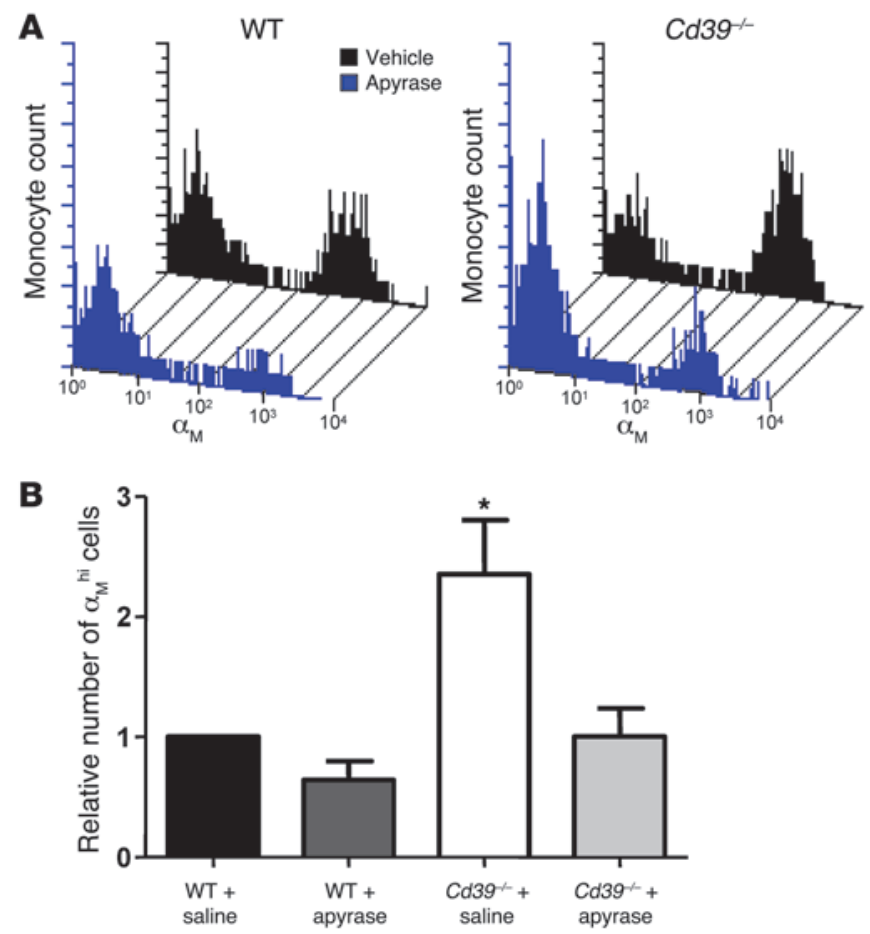

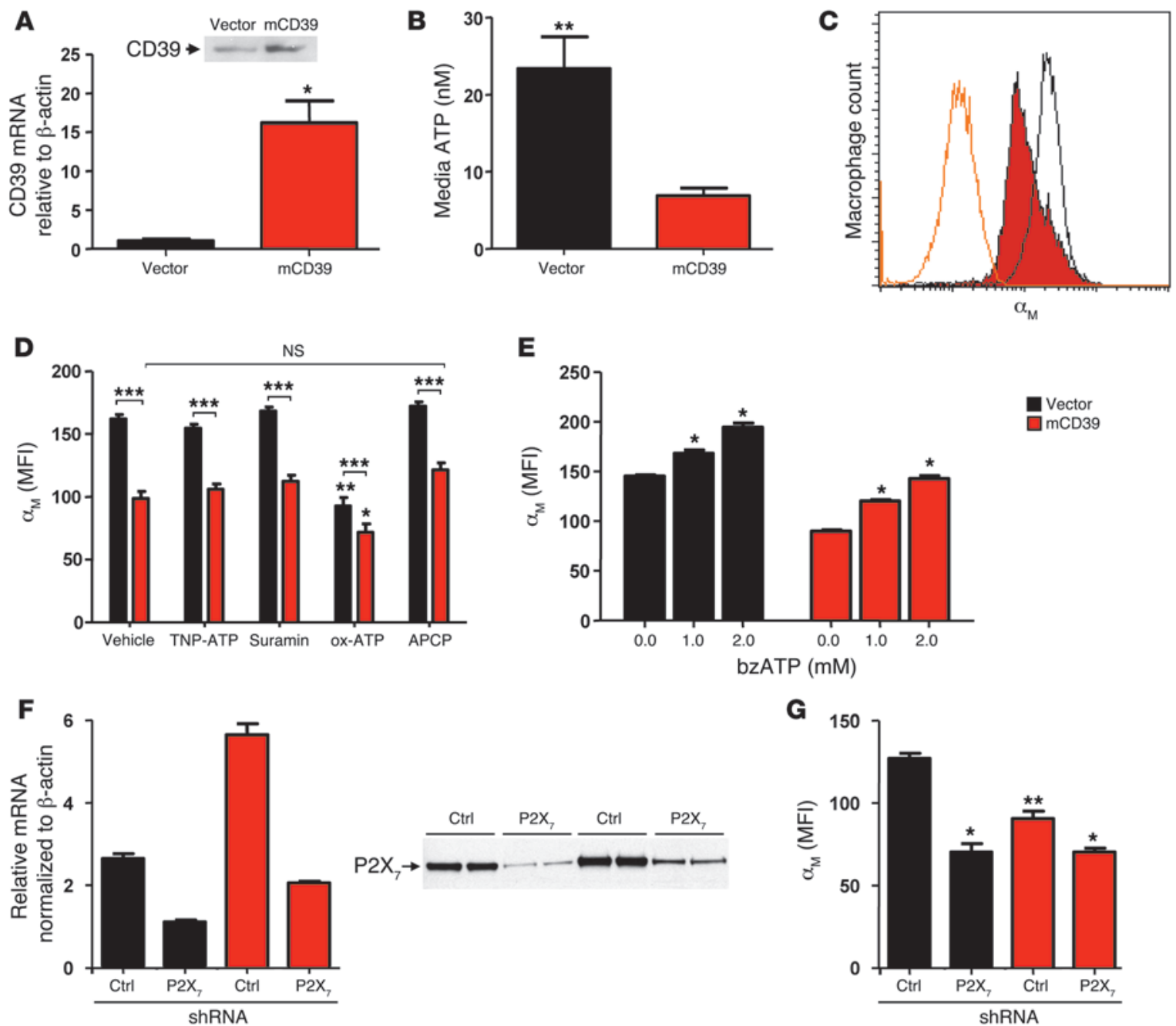

Figure 8

Regulation of $\alpha_{M} \beta_{2}$-integrin in RAW 264.7 macrophages. (A) Relative murine Cd39 mRNA expression was measured using RT-PCR in empty vector-transfected and $\mathrm{mCD} 39$-overexpression vector-transfected macrophages. A representative immunoblot of membrane protein is shown. (B) Free ATP was measured in the medium of each cell line to assess the effect of altered CD39 expression on ambient ATP. (C) Representative histograms of $\alpha_{M}$ expression in empty vector-transfected (black overlay), mCD39-transfected (red) macrophages, and isotype control (orange overlay). (D) Empty vector- and mCD39 vector-transfected macrophages were modulated pharmacologically to determine the contribution of various $\mathrm{P} 2$ receptors and adenosine formation in the regulation of $\alpha_{M} \beta_{2}$-integrin. (E) bzATP, a specific $\mathrm{P} 2 \mathrm{X}_{7}$ receptor agonist, was used to treat macrophages to determine modulation of $\alpha_{M} \beta_{2}$-integrin. $(F)$ Relative P2X $X_{7}$ receptor mRNA was measured by quantitative PCR in macrophage cell lines that expressed either vector or $\mathrm{mCD}_{3} 9$ as well as either control shRNA or shRNA targeting the $\mathrm{P}_{2} \mathrm{X}_{7}$ receptor. A representative $\mathrm{P} 2 \mathrm{X}_{7}$ receptor immunoblot of membrane protein is shown. $(\mathbf{G}) \alpha_{M}$ expression following modulation of CD39 expression, $P 2 X_{7}$ receptor expression, or both. $n=6$ per group; ${ }^{\star} P<0.01$ versus all other groups, ${ }^{\star \star} P<0.001$ versus all other groups, ${ }^{\star \star \star} P<0.001$.

apyrase resulted in an approximately $40 \%$ reduction in the number of $\alpha_{M} \beta_{2}$-integrin high expressers relative to vehicle.

CD39 overexpression reduces cell-surface $\alpha_{M} \beta_{2}$-integrin expression. We transfected RAW 264.7 murine macrophages with either empty pCDNA3.1 vector or PCDNA3.1 containing murine CD39 to explore the relationship between CD39 and $\alpha_{M} \beta_{2}$-integrin in vitro. Stable CD39 transfectants expressed 15-fold more Cd39 mRNA than vector control cells, with a concurrent increase in membrane CD39 protein (Figure 8A). After media exchange with serum-free media, RAW cells overexpressing CD39 were found to have $71 \%$ less ATP in their media when compared with vector transfectants, likely reflecting the difference in CD39 protein expression (Figure 8B). The source of this ATP was presumed to be leakage or release from the macrophages themselves. When the CD39-overexpressing cells were analyzed by flow cytometry, they maintained a resting state with $40 \%$ less cell surface $\alpha_{M} \beta_{2}$-integrin than empty vector transfectants (Figure 8C). We hypothesized that basal ATP released from cells was metabolized more efficiently in CD39-overexpressing cells. This implies that the reduced $\alpha_{M} \beta_{2}$-integrin expression phenotype results from either reduced stimulation of $\mathrm{P} 2$ receptors or increased adenosine generation. To distinguish between these two possibilities, we used adenosine $5 '$-( $\alpha, \beta$-methylene)diphosphate (APCP), a specific CD73 inhibitor, to block conversion of CD39-generated AMP into adenosine by CD73 $(19,29)$. In the presence of APCP, only a small, insignificant increase in the expression of $\alpha_{M} \beta_{2}$-integrin was observed, imply- 


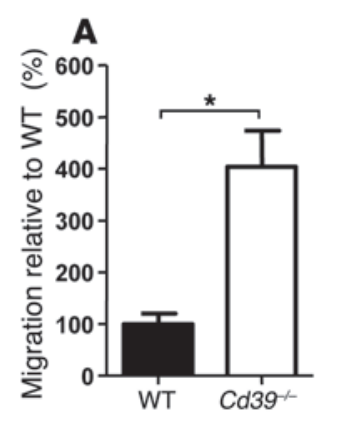

B
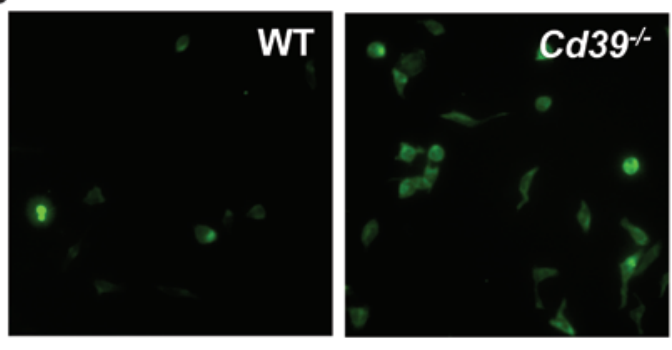

E

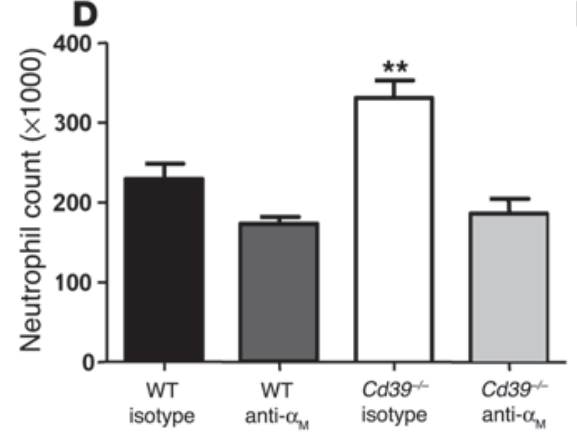

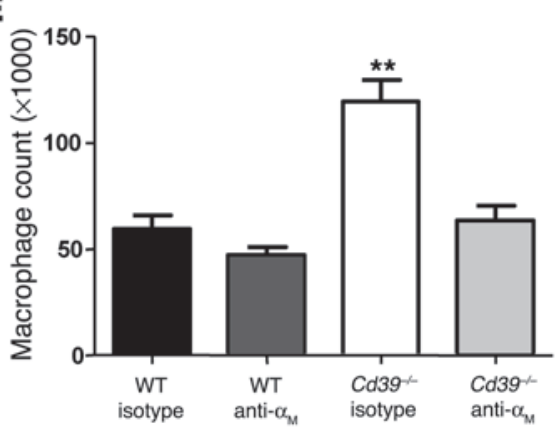

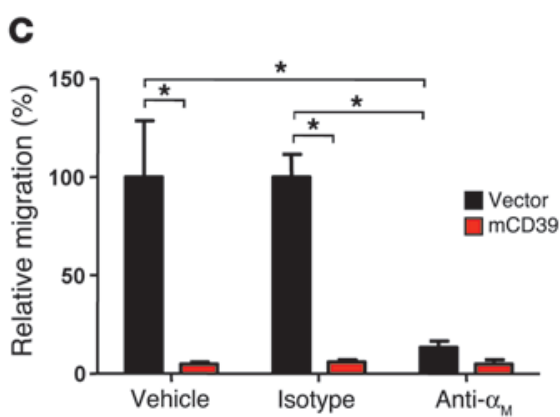

$\mathbf{F}$

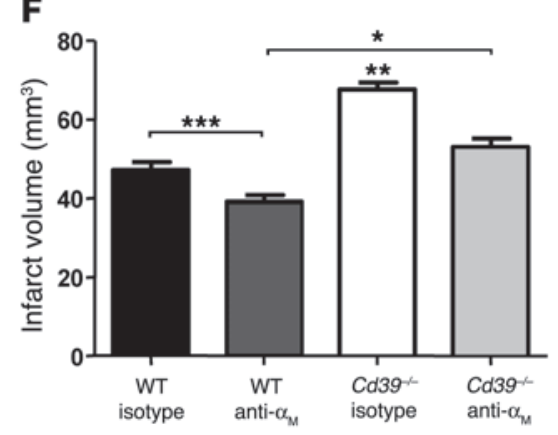

Figure 9

CD39 regulates leukocyte trafficking via $\alpha_{M} \beta_{2}$-integrin in vitro and in vivo. (A) Transmigration of WT or Cd39-/- peritoneal macrophages on fibrin(ogen)-coated Transwells. (B) Representative microscope field of transmigrated primary macrophages stained with F4/80 acquired with a $20 \times$ objective $(0.325 \mu \mathrm{m} /$ pixel). (C) Transmigration on fibrin(ogen)-coated Transwells was assessed using RAW 264.7 macrophages transfected with mCD39 or control vector. Some wells were treated with $\alpha_{M}$ functional blocking antibody or an isotype control. Migration was quantified relative to vehicle-treated, vector-transfected macrophages. In vivo studies examined leukocyte sequestration in WT and Cd39-/- mice treated with an $\alpha_{M} \beta_{2}$-integrin functional blocking antibody 48 hours after induction of cerebral ischemia. Flow cytometry was used to assess neutrophil (D) and macrophage (E) infiltration. (F) Quantification of MRI of infarcted ischemic hemispheres of mice treated with isotype control antibody or $\alpha_{M}$-integrin functional blocking antibody. $n=3$ per group in $\mathbf{A}$ and $\mathbf{B}, n=9$ per group in $\mathbf{C}, n=7$ per group in $\mathbf{D}-\mathbf{F}$. ${ }^{\star} P<0.001$ between indicated groups; ${ }^{* \star} P<0.001$ versus all other groups; ${ }^{* *} P<0.05$ between indicated groups.

ing that this phenotype is not dependent upon the generation of adenosine (Figure 8D).

As the suppression of $\alpha_{M} \beta_{2}$-integrin expression by CD39 appears to be independent of adenosine generation, experiments were next directed toward elucidating a role for ambient ATP. ATP binds to and activates purinergic receptors. To investigate a direct role for purinergic receptor engagement by adenine nucleotide phosphates, we blocked purinergic signaling using the inhibitors suramin (which inhibits $\mathrm{P} 2 \mathrm{X}_{1-3,5}$ and $\left.\mathrm{P}_{2} \mathrm{Y}_{1,11}\right)(4,30-32), 2^{\prime}, 3^{\prime}-O-(2,4,6-$ trinitrophenyl) ATP (TNP-ATP; which inhibits $\left.\mathrm{P}_{2} \mathrm{X}_{1-4}\right)(33,34)$, or periodate oxidized ATP (ox-ATP; which inhibits $\left.\mathrm{P}_{2} \mathrm{X}_{7}\right)(35,36)$. Little change in $\alpha_{M} \beta_{2}$-integrin expression was seen in either CD39overexpressing or vector-transfected cells when treated with suramin or TNP-ATP. However, strong suppression of $\alpha_{M} \beta_{2}$-integrin expression was conferred by the specific $\mathrm{P}_{2} \mathrm{X}_{7}$ inhibitor ox-ATP (Figure 8D). Blockade of the $\mathrm{P} 2 \mathrm{X}_{7}$ receptor in vector-transfected macrophages brought $\alpha_{M} \beta_{2}$-integrin expression to levels lower than those of CD39-overexpressing macrophages. Not surprisingly, as CD39 dissipates ATP and hence itself should indirectly reduce $\mathrm{P}_{7}$ receptor stimulation, $\mathrm{P} 2 \mathrm{X}_{7}$ receptor blockade had a significant but smaller effect on $\alpha_{M} \beta_{2}$-expression in mCD39 transfectants. Finally, the specific $\mathrm{P}_{2} \mathrm{X}_{7}$ receptor agonist $2^{\prime}\left(3^{\prime}\right)$-O-(4-benzoylbenzoyl)ATP (bzATP) dose-dependently induced $\alpha_{M} \beta_{2}$-integrin expression in both cell lines (Figure 8E). This suggested that suppression of $\alpha_{M} \beta_{2}$-integrin expression in CD39 transfectants is due to diminished $\mathrm{P} 2 \mathrm{X}_{7}$ receptor stimulation in CD39-overexpressing cells.
To confirm that the changes in integrin expression were not due to pharmacologically induced off-target effects, we employed a gene-silencing approach. By use of an shRNA that targeted the $\mathrm{P} 2 \mathrm{X}_{7}$ receptor as well as an empty vector control, the $\mathrm{P} 2 \mathrm{X}_{7}$ receptor was silenced at mRNA and protein levels in both control and CD39-overexpressing macrophage cell lines (Figure 8F). These cell lines were subsequently examined by flow cytometry for $\alpha_{M} \beta_{2}$-integrin expression. The cells each carried 2 plasmids, one containing mCD39 or corresponding control vector; the other, $\mathrm{P}_{2} \mathrm{X}_{7}$ shRNA or corresponding control vector. These experiments confirmed the earlier transfection experiments shown in Figure 8C; i.e., when the single transfectants were additionally transduced with a control shRNA, overexpression of $\mathrm{mCD} 39$ still resulted in a significant diminution of $\alpha_{\mathrm{M}}$-integrin expression. In contrast, transduction with a P2 $\mathrm{X}_{7}$-silencing shRNA completely abolished the difference in integrin expression seen between CD39-overexpressing and control cells (Figure 8G). In essence, $\mathrm{P} 2 \mathrm{X}_{7}$ suppression uncouples CD39 levels from regulation of $\alpha_{M}$-integrin levels. This implies that CD39 can regulate the expression of $\alpha_{M} \beta_{2}$-integrin by dissipating ATP that would otherwise activate the $\mathrm{P} 2 \mathrm{X}_{7}$ receptor.

CD39 regulates leukocyte trafficking via $\alpha_{M} \beta_{2}$-integrin in vitro and in vivo. Our in vitro studies suggest that the amplified leukocyte recruitment in Cd39-null mice is a consequence of upregulated $\alpha_{M} \beta_{2}$-integrin expression in Cd39-null leukocytes. To further explore this, we performed leukocyte transmigration assays on fibrin(ogen)-coated Transwells. Fibrin(ogen) is a known cognate 
binding partner for $\alpha_{M} \beta_{2}$-integrin. In this assay, $C d 39^{-/-}$peritoneal macrophages migrated $300 \%$ more than WT controls (Figure 9, $\mathrm{A}$ and B). Further, CD39-overexpressing macrophages exhibited a greater than $90 \%$ reduction in transmigration compared with control cells (Figure 9C). Antibody blockade of the $\alpha_{M}$ subunit demonstrated that the enhanced leukocyte migration seen in vector-transfected cells was dependent upon $\alpha_{M} \beta_{2}$-integrin. We further sought to determine whether $\alpha_{M} \beta_{2}$-integrin might affect leukosequestration in our cerebral ischemia studies. Cd39-null and WT mice were treated with $\alpha_{\mathrm{M}}$-blocking or isotype control antibody. Isotype-treated $\mathrm{Cd} 39^{-/-}$mice had $61 \%$ more infiltrating neutrophils and $104 \%$ more infiltrating macrophages than isotype-treated WT mice, similar to our prior data (Figure 2) showing increased cerebral leukosequestration after ischemia in untreated $\mathrm{Cd} 39^{-/-}$mice. This demonstrates that the leukocyte infiltration phenotype was not affected by an isotype-matched antibody $(P<0.001$; Figure 9, D and $\mathrm{E})$. In sharp contrast, treatment of ischemic $\mathrm{Cd} 39^{-/-}$mice with $\alpha_{\mathrm{M}}$-blocking antibody yielded a striking decrease in the number of infiltrating macrophages and neutrophils when compared with ischemic Cd39-/- mice treated with isotype control antibody (Figure 9, D and E). Furthermore, antibody blockade in $\mathrm{Cd} 33^{-/-}$mice restored levels of leukocyte trafficking to those seen in isotype antibody-treated WT mice. Analysis of peripheral blood showed that this abrogated leukocyte trafficking was not due to pan-leukodepletion following intravenous antibody administration (data not shown). When antibody-treated mice were examined by MRI, both WT and CD39-deficient mice were significantly protected by treatment with an $\alpha_{\mathrm{M}}$-integrin blocking antibody (Figure $9 \mathrm{~F}$ ). The fact that $\alpha_{M}$ blockade was incompletely protective in $\mathrm{Cd} 39^{-/-}$mice (i.e., $\alpha_{M}$ blockade did not reduce infarct volumes to the extent seen in $\alpha_{M}$-antibody-treated WT mice) suggests that injury mechanisms other than $\alpha_{\mathrm{M}}$-dependent leukosequestration are also at play.

To investigate whether heterotypic monocyte-platelet interactions could be contributing, we measured monocyte-platelet aggregates; there were no significant differences in monocyte-platelet aggregates between WT and $\mathrm{Cd} 39^{-/-}$mice, though there was a trend for there to be more in the CD39-deficient mice (Supplemental Figure 2). Together, these data demonstrate a critical role for $\alpha_{M} \beta_{2}$ integrin-dependent leukosequestration in the tissue injury in ischemic stroke, especially in $\mathrm{Cd} 39^{-/-}$mice.

\section{Discussion}

Disruption of blood flow to metabolically active tissues that depend on an uninterrupted supply of oxygen and other nutrients elicits a sequence of events that can ultimately lead to cell death. The initial injury, triggered by substrate deprivation, is amplified by thrombotic occlusion and infiltration of activated leukocytes (37). Under quiescent conditions, CD39, an ectoapyrase expressed on endothelium and leukocyte subpopulations (38), rapidly metabolizes ADP and ATP released by activated platelets, thereby inhibiting platelet recruitment into an evolving thrombus. To date, this nucleotidase has not been recognized as a modulator of postischemic inflammatory cascades. We herein identify what we believe to be a novel paradigm by which CD39 expressed on the leukocyte surface autoregulates the leukocyte's own ambient nucleotide milieu, thereby controlling its adhesive properties for inflamed vasculature and for circulating cells.

Our previous work demonstrated that CD39-deficient mice developed worse clinical outcomes than CD39-bearing controls following challenge by cerebral ischemia (7). This resulted in increased local platelet activation and thrombus formation in mice lacking CD39. In addition to corroborating previous observations, our new data highlight additional mechanisms by which CD39 protects against postischemic cerebral injury. This adds a new dimension to our understanding of the role of CD39 on leukocytes in regulation of tissue injury in response to ischemic stress. The present studies, using a cell population-specific CD39 deletion strategy, demonstrate a critical role for leukocyte CD39 in regulation of platelet reactivity. This extends previous work in which human CD39 overexpressed in marrow-derived cells enhances the endogenous inhibitory effects of CD39 on platelet reactivity (27).

Earlier investigations could not fully attribute the sequelae of stroke to either the leukocyte or platelet populations, leading to the hypothesis that they may interact in a synergistic manner (2). Our previous data showed that platelet inhibition alone (with GPIIb/IIIa blockade) is incompletely protective in the setting of stroke (2). In addition, CD39 exerts its inhibitory activity at an earlier stage than GPIIb/IIIa (9). Our current data indicate that accumulated leukocytes contribute to ischemic cerebral injury as well. Blockade of $\alpha_{\mathrm{M}}$-integrin is protective in our model of cerebral ischemia in part by preventing leukocyte trafficking. In the CD39-knockout mice, $\alpha_{\mathrm{M}}$-integrin blockade reduced leukosequestration to levels equal to those in WT mice. This suggests that the increased leukosequestration in $C d 39^{-/-}$mice was related to the increased $\alpha_{M} \beta_{2}$-integrin expression on circulating $\mathrm{Cd} 39^{-/-}$leukocytes. When infarct volumes were examined, protection by $\alpha_{\mathrm{M}}$-integrin blockade was incomplete, likely attributable to the fact that mechanisms other than leukosequestration are also in play and contribute to tissue injury in ischemic cerebral infarction. One potential explanation for the incomplete protection afforded by $\alpha_{\mathrm{M}}$-integrin blockade is that thrombosis and inflammation intersect through the formation of leukotrienes (39) and leukocyte-platelet aggregates (40). Though we did not see a statistically significant increase in the percentage of circulating monocytes that were bound to platelets in $\mathrm{Cd} 39^{--}$mice (vs. WT), there was a trend in that direction. The possibility remains that interactions of monocytes and platelets directly at the vascular surface is an important contributory mechanism to ischemic tissue injury. Interestingly, other models of intestinal (41) and renal (42) ischemia revealed a global susceptibility to ischemic injury in Cd39-null animals. In each of these studies, the protective effects of CD39 were attributed to its location on endothelial cells, where it modulated platelet activation and recruitment as well as vascular permeability. Although CD39 expression is recognized on certain leukocyte populations (B cells, monocytes, neutrophils; refs. 38, 43), the present investigation may be the first to definitively attribute resistance to ischemic injury to leukocytes bearing CD39 apyrase.

The current bone marrow transplantation studies demonstrate that CD39 on leukocytes (particularly monocytes) critically regulated leukocyte influx into ischemic tissue, as well as tissue injury itself. In a previous study using a different gene-targeting strategy, Cd39-/- peritoneal macrophages exhibited less invasive potential in Matrigel chemotaxis or tumor models compared with WT controls (44). Our present demonstration of a clear predisposition to enhanced leukocyte sequestration in CD39-deficient mice was derived with a strategy involving deletion of the conserved apyrase domain. However, these 2 models of leukocyte migration are quite different: Ischemia-driven cell infiltration is guided by a well-organized sequence of events leading from cell adhesion to diapedesis, whereas the Matrigel models involve proteolysis of the matrix as well as a potentially different set of diapedesis cues. Nevertheless, 
both Cd39-deletional mutants are susceptible to ischemic injury and both could be rescued by treatment with apyrase (42) or solCD39 $(7,8)$. In general, the protective effects of solCD39 have been attributed to its ability to block platelet activation and recruitment. However, our current study's apyrase treatment experiments demonstrate that the protective effects of this enzyme may also lie in its ability to inhibit leukocyte recruitment to ischemic tissues.

The classic paradigm of inflammatory signaling has focused upon recruitment and activation of leukocytes, with concomitant release of soluble mediators that amplify and accelerate the process. It is possible that some of the antiinflammatory properties of CD39 are related to adenosine generated by the sequential phosphohydrolysis of ATP to ADP, AMP, and adenosine, the terminal reaction being catalyzed by CD73. We examined the role for adenosine generated downstream of CD39 in our in vitro model by using APCP, which blocks the function of CD73, thereby preventing the generation of adenosine from AMP. As there was no difference in macrophage surface integrin expression irrespective of CD73 activity, this suggests that the major contribution of CD39 to suppression of ischemic leukosequestration lies in the dissipation of ADP and ATP rather than downstream generation of adenosine. Given the high abundance of CD39 on the surface of myeloid lineage cells, one can envision leukocytes regulating not only each other's activation, but also their own activation in a cell-autonomous fashion, via catabolism of proinflammatory agonists.

This scheme of catabolism-mediated, autonomous control of activation is seen in CD39's regulation of $\alpha_{M} \beta_{2}$-integrin. Though $\alpha_{M} \beta_{2^{-}}$ integrin is normally found in submembrane, preformed storage granules (45), in CD39-deficient animals, leukocytes appear to have a basal state of heightened $\alpha_{M} \beta_{2}$-integrin expression. One potential explanation for this observation is that ATP, known to upregulate $\alpha_{M} \beta_{2}$-integrin expression $(16,28)$, would be present at higher levels in the microenvironment of $\mathrm{Cd}_{39^{-/-}}$cells due to their lack of ATP phosphohydrolase activity. Although basal levels of plasma nucleotides may not vary between $\mathrm{Cd}_{39^{-/-}}$and WT animals (7), treatment of Cd39-null monocytes with soluble apyrase does restore a WT $\alpha_{M} \beta_{2}$-integrin surface expression pattern, indicative of differences in local concentrations of ATP and ADP, as also shown by downregulation of platelet ADP responses $(7,11)$. This could be important, as extracellular ATP has been shown to play an important role in signaling intracellular events. Extracellular purinergic nucleotides, such as ATP and ADP, are known to activate families of receptors; these include ligand-gated receptors (P2X family) as well as $\mathrm{G}$ protein-coupled receptors (P2Y family). Among the P2X family, there are 7 known receptor subunits that multimerize to form receptors of varying specificities. Our data demonstrate that the homomeric $\mathrm{P} 2 \mathrm{X}_{7}$ receptor regulates expression of $\alpha_{\mathrm{M}} \beta_{2}$-integrin in unstimulated macrophages and that $\alpha_{M} \beta_{2}$-integrin expression in these cells is inversely correlated with cell-surface CD39 (46). This is complementary to previous work, which established a putative role for CD39 in the regulation of the $\mathrm{P} 2 \mathrm{X}_{7}$ receptor on endothelial cells (47). The $\mathrm{P} 2 \mathrm{X}_{7}$ receptor, although not previously linked to integrin expression, is known to play a role in regulation and function of the inflammasome, a multiprotein complex that regulates production and release of proinflammatory cytokines $(6,48)$. Thus, in the setting of ischemia, where ATP and ADP are released as a consequence of tissue destruction, CD39-deficient mice would be less able to metabolically control ATP-driven inflammatory cascades. Cd39-/- leukocytes would become primed for adhesion and diapedesis across an activated endothelium. By contrast, WT leukocytes, at baseline, would be less ready to initiate transmigration and induce subsequent tissue damage. A decrease in $\alpha_{\mathrm{M}}$-integrin expression has already been shown to be profoundly protective in the setting of cerebral ischemia $(37,49)$. Furthermore, the importance of this CD39-mediated integrin regulation is supported by our data showing normalization of leukocyte trafficking in $C d 39^{-/-}$mice by $\alpha_{M} \beta_{2}$-integrin blockade. Thus, CD39 is likely to inhibit leukosequestration via its repression of $\mathrm{P} 2 \mathrm{X}_{7}$-driven $\alpha_{\mathrm{M}} \beta_{2}$-integrin expression. This finding is particularly important given that $\alpha_{M} \beta_{2}$-integrin is a critical adhesion receptor in the formation of leukocyte-platelet heteroaggregates and in light of our previous finding that CD39-deficient mice have heightened platelet deposition in the ischemic brain (7).

In summary, our experimental results demonstrate a direct link between CD39 and control of leukocyte migration into ischemic tissue. This occurs via a previously unrecognized mechanism by which leukocytes regulate their own flux through metabolism of agonists released into local vascular microenvironments by cellular activation or leakage from dying cells. Previously, CD39 has been demonstrated to be the major control system for blood fluidity $(7,9,10)$; these new data add to the pleiotropic properties of CD39 on platelet activation and recruitment, demonstrating that both CD39-bearing leukocyte and endothelial subpopulations can suppress platelet activation. Furthermore, these new data indicate a prime role for CD39 in modulating the expression of glycoprotein adhesive receptors on the surface of leukocytes, particularly cells of the monocyte lineage. Thus, CD39 resides at the critical nexus of inflammation, coagulation, and thrombosis as a vital regulator of vascular homeostasis.

\section{Methods}

Animal experiments were approved by and carried out in accordance with the University of Michigan Institutional Animal Care and Use Committee. All reagents, unless otherwise noted, were obtained from SigmaAldrich. Mice deficient for the Cd39 gene (generated by replacement of the enzymatically active extracellular portion of the CD39 molecule [exons 4-6; apyrase-conserved regions 2-4] with a PGKneo cassette) were used for the indicated experiments (7).

Photothrombotic model of MCA occlusion. Cerebral ischemia was induced as previously described (20) by an operator blinded to experimental conditions. The experiment was performed in both untransplanted and transplanted mice aged 15-16 weeks. In brief, mice were anesthetized with ketamine/ xylazine; body temperature was maintained at $37^{\circ} \mathrm{C}$ using a heating pad. The skin overlaying the calvaria was incised, exposing the temporalis muscle. The temporalis was incised, after which a burr hole was drilled to visualize the MCA. The exposed MCA was placed under a 542-nm neon laser, and the mouse was injected intravenously with rose bengal dye $(1 \mathrm{mg} / 25 \mathrm{~g}$ body weight $)$ dissolved in normal saline $(10 \mathrm{mg} / \mathrm{ml})$. MCA blood flow was monitored with a laser Doppler flow probe (Transonic Systems Inc.) to determine occlusion, defined as a greater than $80 \%$ reduction in blood flow sustained for 10 minutes. After occlusion, the laser and flow probe remained in place for an additional 15 minutes, after which the probe was removed, the skin incision closed, and the mice allowed to recover.

MRI. Forty-eight hours after induction of cerebral ischemia, infarct volumes were assessed by MRI. Mice were anesthetized with a $2 \%$ isoflurane/air mixture throughout MRI examination. Mice were placed prone in a $7.0 \mathrm{~T}$ Varian MR scanner (183-mm horizontal bore), with body temperature maintained at $37^{\circ} \mathrm{C}$ using circulated heated air. A double-tuned volume radio-frequency coil was used to scan the head region of the mice. Axial $\mathrm{T}_{2}$-weighted images were acquired using a spin-echo sequence with the following parameters: repetition time (TR)/effective echo time (TE), 4,000/40 $\mathrm{ms}$; field of view, $30 \times 30 \mathrm{~mm}$; matrix, $128 \times 128$; slice thickness, $0.5 \mathrm{~mm}$; 
slice spacing, $0 \mathrm{~mm}$; number of slices, 25; and number of scans, 1 (total scan time, $\sim 8$ minutes). Infarct volumes for each animal were quantified by an observer blinded to experimental conditions.

Neurologic deficit scoring. Evaluations were made by an operator blinded to experimental conditions using a previously described 5-point scoring system (50): 1 , normal motor function; 2 , flexion of the torso and contralateral forelimb upon lifting of the animal by the tail; 3 , circling to the contralateral side with normal posture at rest; 4 , leaning to the contralateral side at rest; 5 , no spontaneous motor activity.

Immunohistochemistry of ischemic brains. Contralateral and ischemic hemispheres of infarcted mice were fixed in $4 \%$ paraformaldehyde and embedded in Tissue-Tek OCT (Sakura Finetek) prior to sectioning. Neutrophils were identified by their expression of LY-6G (SouthernBiotech). Macrophages were identified by their expression of F4/80 (CALTAG Laboratories, Invitrogen). Primary antibody was detected with a VECTASTAIN ABC-Peroxidase Kit (Vector Laboratories) and developed using a TSA Fluorescein Tyramide Reagent Pack (Perkin-Elmer). Nuclei were stained with DAPI. Images were acquired with a $4 \times$ objective $(1.614 \mu \mathrm{m} /$ pixel $)$ using MetaMorph version 7.0r3 software (Molecular Devices) on an Eclipse TE2000-E microscope (Nikon Instruments Inc.).

Flow-cytometric analysis of infiltrating leukocyte populations. Upon euthanasia, the cerebrum was removed and divided into ischemic and nonischemic hemispheres. The hemispheres were mechanically dissociated with a scalpel and an 18-gauge syringe to yield single-cell suspensions. A Percoll gradient (density, $1,130 \mathrm{~g} / \mathrm{ml}$ ) (GE Healthcare) was used to separate the infiltrating cell population from myelin and neuronal debris. Erythrocyte contaminants were lysed using FACS Lyse (BD) per the manufacturer's protocol. Infiltrating cells were counted using a hemocytometer prior to flow-cytometric analysis on a FACSCalibur (BD) using CellQuest software (BD). Viable cells were identified by the absence of propidium iodide (PI; BD) staining. Nonspecific antibody interactions were inhibited using Fc Block (BD). Leukocytes were initially identified as PE-conjugated $\mathrm{CD} 45^{\mathrm{hi}}(\mathrm{BD})$. CD $45^{\text {hi }}$ cells were further identified as neutrophils based on FITC-conjugated LY-6G (BD) positivity and macrophages by FITC-conjugated F4/80 (Serotec) positivity. Ten thousand cells from the cellular fraction of each hemisphere were acquired per data set.

TLC analysis of leukocyte AT(D)Pase activities. Mouse blood was drawn from the retro-orbital plexus into heparinized capillaries and was subsequently layered onto Histopaque 1119 (Sigma-Aldrich) before centrifugation according to the manufacturer's protocol. Purified buffy coats were washed and resuspended in RPMI 1640 (GIBCO, Invitrogen) containing $5 \mathrm{mM} \beta$-glycerophosphate (Sigma-Aldrich) (51). For assessment of enzymatic function, cell suspensions of either CD39-deficient or WT leukocytes were mixed $1: 1$ with $1.0 \mathrm{mM}\left[8-{ }^{14} \mathrm{C}\right] \mathrm{ADP}$ (PerkinElmer) or $1.0 \mathrm{mM}$ $\left[8-{ }^{-14} \mathrm{C}\right]$ ATP (MP Biomedicals) and incubated at $37^{\circ} \mathrm{C}$ for 30 minutes. The incubation time was chosen to ensure linearity of the reaction with time, i.e., a maximum of $30 \%$ of the initially introduced substrate was metabolized. The reaction was stopped using $8 \mathrm{M}$ formic acid (52). The reaction mixture was spotted on silica gel/TLC plates (Fluka, Invitrogen) along with a ladder (a mixture of $\left[8-{ }^{14} \mathrm{C}\right] \mathrm{AMP}$ [GE Healthcare], $\left[8-{ }^{-14} \mathrm{C}\right] \mathrm{ADP}$, and $\left[8-{ }^{14} \mathrm{C}\right] \mathrm{ATP}$ ). The nucleotides were separated by TLC with isobutanol/isoamyl alcohol/ 2-ethoxyethanol/ammonia/ $\mathrm{H}_{2} \mathrm{O}$ (9:6:18:9:15) as previously described (10, 51). TLC plates were then exposed to a phosphoimaging screen (Kodak) and analyzed using a Typhoon Trio $^{+}$(GE Healthcare).

Apyrase rescue of CD39-deficient mice. Cd39-null mice were treated with either vehicle or grade VII potato apyrase $(4 \mathrm{U} / \mathrm{g}) 1$ hour prior to induction of cerebral ischemia. This dose has been used previously to rescue Cd39-null mice (11). Three hours later, mice were treated a second time with either vehicle or apyrase. After 48 hours, mice were examined via either MRI or flow cytometry.

Bone marrow transplantation. At 7 weeks of age, Cd39-/- and WT mice underwent bone marrow transplantation as previously described (53). In brief, mice were myeloablated via a 12.5-Gy radiation exposure administered in 2 doses 3 hours apart. Mice were reconstituted with approximately $4 \times 10^{6}$ BMDCs administered intravenously as follows: Cd39-deficient mice were reconstituted with $\mathrm{Cd} 39^{-/-}$bone marrow (to generate global knockout controls) or with WT bone marrow (to generate mice with "tissue-only CD39 ablation"). WT mice were reconstituted with WT bone marrow (to generate WT controls), or Cd39-/- bone marrow (to generate mice with CD39 in established tissue but not in BMDCs ["leukocyte CD39 ablation"]). These chimeras are referred to as $\mathrm{KO} \rightarrow \mathrm{KO}, \mathrm{WT} \rightarrow \mathrm{KO}, \mathrm{WT} \rightarrow \mathrm{WT}$, and $\mathrm{KO} \rightarrow \mathrm{WT}$, respectively. Mice were allowed to recover for 8-10 weeks prior to stroke induction or phenotype examination.

Chimerism assay. Citrated murine blood $(150 \mu \mathrm{l})$ was drawn from the retroorbital plexus of chimeric mice and the leukocyte fraction isolated using a Histopaque 1099 (Sigma-Aldrich) density gradient according to a method previously described for human blood $(16,28)$. Cells were subsequently sorted based on their characteristic forward- and side-scatter properties to identify monocyte and neutrophil populations (54). Neutrophils were secondarily gated for LY-6G positivity. Sorted monocyte and neutrophil populations were then collected and stained using HEMA 3 (Fisher Scientific) to confirm leukocyte purity (Supplemental Figure 1). Other collected cells were kept for DNA isolation. Probe and primer sets targeting both the neomycin cassette present in $\mathrm{Cd} 39^{-/-}$mice and NGF were used in a duplex format. The probes and primers were synthesized by Applied Biosystems with the following sequences: neomycin probe: 5 '-CATCGCATCGAGCGAGCACGTACT-3'; neomycin primer 1: 5'-CGGCTGCATACGCTTGAT-3'; neomycin primer 2: 5'-CGACAAGACCGGCTTCCAT-3'; NGF probe: 5'-ACGGTTCTGCCTGTACGCCGATCA-3'; NGF primer 1: 5'-TGCATAGCGTAATGTCCATGTTG-3'; NGF primer 2: 5'-TCTCCTTCTGGGACATTGCTATC-3' $(55,56)$. DNA from purified leukocytes was amplified with the above probe and primer sets using TaqMan Gene Expression Master Mix (Applied Biosystems) in an ABI Prism7000 Sequence Detection System.

Whole-blood analysis of cell-surface CD39 expression in bone marrow-reconstituted mice. Citrated murine blood $(150 \mu \mathrm{l})$ was drawn from the retro-orbital plexus of bone marrow-reconstituted mice and sorted into neutrophil and monocyte populations as described above. In addition to LY-6G, these cell populations were concurrently stained for PI, to exclude dead cells, and PEconjugated CD39 (eBioscience) before analysis by flow cytometry.

Analysis of CD39 expression in tissue homogenates. Whole lungs of bone marrow-reconstituted mice were digested in $0.1 \%$ collagenase B (Roche Applied Science) prior to dissociation with a gentleMACS Dissociator (Miltenyi Biotec). Cells were divided $10^{6}$ per tube before staining with: FITC-conjugated CD31 (BD), PE-conjugated CD39 (eBioscience), APC-conjugated CD45 (BD) to identify CD31 ${ }^{+} \mathrm{CD} 45^{-}$endothelial cells; FITC-conjugated LY-6G (BD), PE-conjugated CD39 (eBioscience), APC-conjugated CD45 (BD) to identify $\mathrm{LY}-6 \mathrm{G}^{+} \mathrm{CD} 45^{+}$resident tissue neutrophils; or FITC-conjugated CD45, PEconjugated CD39 (eBioscience), APC-conjugated F4/80 (Serotec) to identify $\mathrm{F} 4 / 80^{+} \mathrm{CD} 45^{+}$resident tissue macrophages. For each of these stains, 7 -aminoactinomycin D (7-AAD) was used to exclude dead cells (BD).

Whole-blood aggregometry. Heparinized murine blood was drawn from the retro-orbital plexus of mice 2 weeks after bone marrow reconstitution. Whole-blood impedance aggregometry was then performed using a Chrono-log 560CA with the Aggro/Link 810. Whole blood was diluted 4-fold with physiologic saline before stimulation with $2 \mu \mathrm{M}$ ADP (Chrono-log).

Whole-blood analysis of $\alpha_{M} \beta_{2}$-integrin surface expression. Heparinized murine blood (600-800 $\mu \mathrm{l})$ was drawn from the inferior vena cava and the leukocyte fraction isolated using a Histopaque 1099 density gradient according to a method previously described for human blood $(16,28)$. A subset of mice was injected intravenously with either vehicle (saline) or apyrase $(2 \mathrm{U} / \mathrm{g}) 2$ hours prior to blood isolation. After centrifugation, cells were washed with HBSS (GIBCO, Invitrogen), prior to cell labeling with PI, Fc 
block (BD), anti-CD11b (the $\alpha_{M}$ subunit of $\alpha_{M} \beta_{2}$-integrin) Ab conjugated to FITC (BD), or anti-LY-6G Ab conjugated to PE (BD) $\left(15\right.$ minutes, $\left.37^{\circ} \mathrm{C}\right)$. Neutrophils were identified by LY-6G positivity and their forward- and side-scatter properties. Monocytes were identified by their characteristic forward- and side-scatter properties (54).

Overexpression of CD39 in RAW 264.7 macrophages. RAW 264.7 macrophages were transfected with murine CD39 or vector control and clonally selected as an in vitro correlate to the whole-blood studies. The CD39 open reading frame of full-length mouse CD39 (mCD39) cDNA (ATCC) was PCR amplified using as $5^{\prime}$-primer 5 '-aaaagGtAcccettatggaagatataaagg- $3^{\prime}$ and as $3^{\prime}$-primer, 5'-gccgctcGAgctatactgcctctttccag-3'. This was subcloned into the pCDNA3.1 overexpression vector (Invitrogen). RAW cells were transfected with either the CD39 overexpression construct or empty pCDNA3.1 vector (control) using SuperFect Transfection Reagent (QIAGEN). The day before transfection, RAW cells were plated on 6-well plates (Corning) with $1 \times 10^{6}$ cells per well and grown in RPMI 1640 (Invitrogen) containing $10 \%$ FCS (Invitrogen) $\left(37^{\circ} \mathrm{C}\right.$, humidified atmosphere containing $\left.5 \% \mathrm{CO}_{2}\right)$ until they reached $50 \%-80 \%$ confluence. Transfection complexes were generated using $2 \mu \mathrm{g}$ of mCD39 overexpression plasmid and $10 \mu \mathrm{l}$ of SuperFect in $100 \mu \mathrm{l}$ of serum-free RPMI 1640 medium. The SuperFect mixture was incubated (room temperature, 10 minutes), after which the complexes were added to RAW cells ( $0.6 \mathrm{ml}$ RPMI 1640 medium containing 10\% FBS). After incubating $\left(37^{\circ} \mathrm{C}, 5 \% \mathrm{CO}_{2}, 3\right.$ hours), cells were washed 3 times with HBSS (Invitrogen) and then incubated with RPMI with 10\% FBS. After 72 hours, transfected cells were selected using hygromycin $(300 \mu \mathrm{g} / \mathrm{ml})$.

Membrane protein isolation. RAW cell transfectants were washed twice with PBS (GIBCO, Invitrogen), and membrane protein was isolated as previously described (57). In brief, $2 \mathrm{ml}$ ice-cold hypotonic lysis buffer ( $50 \mathrm{mM}$ sucrose, 10 mM HEPES, pH 7.4, with $1 \mu \mathrm{g} / \mathrm{ml}$ aprotinin, $1 \mu \mathrm{g}$ leupeptin, $1 \mathrm{mM}$ PMSF) was added to each plate before cells were scraped from the dish bottom. Cells were homogenized by 25 strokes of a Dounce homogenizer (Kontes Glass, Kimble Chase) with a tight pestle. After the addition of $132 \mu$ l of $65 \%$ sucrose (w/w; in $10 \mathrm{mM}$ HEPES, pH 7.4), $4 \mu \mathrm{l}$ of $0.5 \mathrm{M} \mathrm{MgCl}_{2}$ into $2 \mathrm{ml}$ of hypotonic lysis buffer, the homogenate was subjected to two 20 -minute spins at $2,000 \mathrm{~g}$ at $4^{\circ} \mathrm{C}$ to pellet nuclei, mitochondria, and unlysed cells. Crude membranes were pelleted from the resulting supernatant by centrifugation at $100,000 \mathrm{~g}$ for 30 minutes at $4^{\circ} \mathrm{C}$, washed quickly in $2 \mathrm{ml}$ of hypotonic lysis buffer, and resuspended in the same buffer. The membranes were flash frozen in aliquots and stored at $-80^{\circ} \mathrm{C}$. Protein concentration was determined by Bio-Rad protein assay.

Free ATP measurement. RAW cell transfectants were washed 2 times with phenol red-free RPMI 1640 (Invitrogen) and then serum-starved for 24 hours. Cell-free medium was then analyzed for endogenous ATP using an ATP bioluminescent assay kit (Sigma-Aldrich). Bioluminescent activity was measured using a VICTOR Light luminometer (PerkinElmer).

$\alpha_{M} \beta_{2}$-integrin expression in transfected RAW 264.7 macrophages. RAW cell transfectants were serum-starved overnight prior to stimulation for 5 hours with TNP-ATP $(33,34), 50 \mu \mathrm{M}$ suramin $(4,30-32), 50 \mu \mathrm{M} \operatorname{APCP}(19,29)$, or $150 \mu \mathrm{M}$ ox-ATP $(35,36)$. Cells were treated ( 30 minutes) with bzATP, a specific $\mathrm{P} 2 \mathrm{X}_{7}$ receptor agonist. Macrophages were analyzed by flow cytometry after staining with an antibody directed against $\alpha_{M} \beta_{2}$-integrin.

Silencing the P2X, receptor in transfected RAW 264.7 macrophages. RAW cells transfected with murine $C d 39$ or the control vector pcDNA3.1 were infected with lentivirus containing either an shRNA targeting the $\mathrm{P}_{2} \mathrm{X}_{7}$ receptor or the control silencing vector pLKO.1. The sequence of the P2X $\mathrm{X}_{7}$ shRNA construct within the pLKO.1 vector was 5'-CCGGGTCTTGCACATGATCGTCTTTCTCGAGAAAGACGATCATGTGCAAGACTTTTG-3'. Both constructs were purchased as lentiviral particles from Sigma-Aldrich. Macrophages were subsequently selected with puromycin to kill nontransfected cells. After 24 hours of serum starvation, each of the 4 cell lines was analyzed by flow cytometry for cell-surface $\alpha_{M} \beta_{2}$-integrin expression.
Macrophage transmigration assay. Primary peritoneal macrophages were elicited by injecting $3 \mathrm{ml}$ of $4 \%$ thioglycollate medium (Sigma-Aldrich) into WT and $C d 39^{---}$mice. Five days later, peritoneal macrophages were isolated via peritoneal lavage and rinsed of debris with PBS. Primary macrophages were then used in a previously described transmigration assay (58). Cells in serum-free RPMI 1640 were added onto the upper well of a 24-well Transwell plate (5- $\mu$ m pore; Costar, Corning) that had RPMI 1640 with $5 \%$ FBS in the lower well. The inserts were precoated on both sides with fibrinogen (100 $\mathrm{mg} / \mathrm{ml}$ ) and then blocked in BSA. After 12 hours at $37^{\circ} \mathrm{C}$, the number of cells that had transmigrated into the lower well was determined. The identity of transmigrating macrophages was confirmed by staining for F4/80. Images were acquired with a $20 \times$ objective $(0.325 \mu \mathrm{m} /$ pixel $)$ using MetaMorph version 7.0r3 software (Molecular Devices) on an Eclipse TE2000-E microscope (Nikon Instruments Inc.). RAW cell transfectants were serum-starved in RPMI 1640 for 24 hours prior to being used in the same transmigration assay. Additional wells were simultaneously treated with either $\alpha_{M} \beta_{2}$-integrin functional blocking antibody (M1/70) or rat IgG2b, $\mathrm{\kappa}$ isotype control antibody, after which the cells were allowed to transmigrate as before.

In vivo $\alpha_{M} \beta_{2}$-integrin blockade. An $\alpha_{\mathrm{M}} \beta_{2}$-integrin functional blocking antibody (M1/70) or rat IgG2b, $\kappa$ isotype control antibody was diluted in saline and administered intravenously $(2 \mathrm{mg} / \mathrm{kg}) 1$ hour prior to induction of cerebral ischemia (59). Additional antibody $(1 \mathrm{mg} / \mathrm{kg})$ was administered 3 and 24 hours after ischemic induction (59). Antibody preparations used were sodium azide-free and contained low endotoxin (NA/LE) to minimize cytotoxicity. Single-cell suspensions derived from ischemic and nonischemic brain hemispheres from antibody-treated mice were analyzed by flow cytometry as described above, 48 hours after ischemic induction. Additional antibody-treated mice were analyzed by MRI as described above.

Statistics. Values are expressed as mean \pm SEM, with the numbers of experiments performed provided in the figure legends. For experiments in which 2 variables were compared, 2-tailed unpaired Student's $t$ tests were used. For experiments in which more than 2 variables were compared, 1-way ANOVA was used, with Fisher's exact test used to test for significant differences. For experiments involving a nominal variable and a measurement variable, the Mann-Whitney $U$ test was used for nominal variables with 2 values, while the Kruskal-Wallis test was used for nominal variables with 3 or more values. Data were considered significantly different when $P$ was less than 0.05 .

\section{Acknowledgments}

The authors thank Mithun Neral for his expert technical assistance. This work was supported by: American Heart Association predoctoral fellowship N009520 (to M.C. Hyman); NIH grants HL086676 (to D.J. Pinsky), NS041460 (to D.J. Pinsky), HL69448 (to D.J. Pinsky), T32 HL007853 (to S.H. Visovatti and D.J. Pinsky), and P01HL089407 (to D.A. Lawrence and D.J. Pinsky); the J. Griswold Ruth MD \& Margery Hopkins Ruth Professorship (to D.J. Pinsky); and the A. Alfred Taubman Medical Research Institute at the University of Michigan; as well as NIH grants HL47073 (to A.J. Marcus) and HL46403 (to A.J. Marcus) at Weill Cornell Medical College, a Merit Review Grant from the Department of Veterans Affairs (to A.J. Marcus), and the Cancer Research and Treatment Fund (to A.J. Marcus).

Received for publication June 9, 2008, and accepted in revised form February 18, 2009.

Address correspondence to: Matthew C. Hyman, 7240 Medical Science Research Building III, 1150 West Medical Center Drive, Ann Arbor, Michigan 48109, USA; Phone: (734) 936-7605; Fax: (734) 936-8266; E-mail: mchyman@umich.edu. 
1. Huang, J., et al. 1999. Neuronal protection in stroke by an sLex-glycosylated complement inhibitory protein. Science. 285:595-599.

2. Choudhri, T.F., et al. 1998. Reduced microvascular thrombosis and improved outcome in acute murine stroke by inhibiting GP IIb/IIIa receptor-mediated platelet aggregation. J. Clin. Invest. 102:1301-1310.

3. Salmi, M., and Jalkanen, S. 2005. Cell-surface enzymes in control of leukocyte trafficking. Nat. Rev. Immunol. 5:760-771.

4. Ralevic, V., and Burnstock, G. 1998. Receptors for purines and pyrimidines. Pharmacol. Rev. 50:413-492.

5. Di Virgilio, F., et al. 2001. Nucleotide receptors: an emerging family of regulatory molecules in blood cells. Blood. 97:587-600.

6. Khakh, B.S., and North, R.A. 2006. P2X receptors as cell-surface ATP sensors in health and disease. Nature. 442:527-532.

7. Pinsky, D.J., et al. 2002. Elucidation of the thromboregulatory role of CD39/ectoapyrase in the ischemic brain. J. Clin. Invest. 109:1031-1040.

8. Belayev, L., et al. 2003. Neuroprotective effect of SolCD39, a novel platelet aggregation inhibitor, on transient middle cerebral artery occlusion in rats. Stroke. 34:758-763.

9. Marcus, A.J., et al. 1997. The endothelial cell ectoADPase responsible for inhibition of platelet function is CD39. J. Clin. Invest. 99:1351-1360.

10. Marcus, A.J., et al. 1991. Inhibition of platelet function by an aspirin-insensitive endothelial cell ADPase. Thromboregulation by endothelial cells. J. Clin. Invest. 88:1690-1696.

11. Enjyoji, K., et al. 1999. Targeted disruption of cd39/ ATP diphosphohydrolase results in disordered hemostasis and thromboregulation. Nat. Med. 5:1010-1017.

12. Zimmermann, H. 1999. Nucleotides and cd39: principal modulatory players in hemostasis and thrombosis. Nat. Med. 5:987-988.

13. Chen, Y., et al. 2006. ATP release guides neutrophil chemotaxis via P2Y2 and A3 receptors. Science. 314:1792-1795.

14. Gorini, S., and la Sala, A. 2008. Hydrolysis of extracellular ATP and immune suppression: humans versus mice. Blood. 111:964-965.

15. Linden, J. 2006. Cell biology. Purinergic chemotaxis. Science. 314:1689-1690.

16. Freyer, D.R., Boxer, L.A., Axtell, R.A., and Todd, R.F., 3rd. 1988. Stimulation of human neutrophil adhesive properties by adenine nucleotides. J. Immunol. 141:580-586.

17. Eltzschig, H.K., et al. 2003. Coordinated adenine nucleotide phosphohydrolysis and nucleoside signaling in posthypoxic endothelium: role of ectonucleotidases and adenosine A2B receptors. J. Exp. Med. 198:783-796.

18. Thompson, L.F., et al. 2004. Crucial role for ecto5 '-nucleotidase (CD73) in vascular leakage during hypoxia. J. Exp. Med. 200:1395-1405.

19. Eltzschig, H.K., et al. 2004. Endogenous adenosine produced during hypoxia attenuates neutrophil accumulation: coordination by extracellular nucleotide metabolism. Blood. 104:3986-3992.

20. Su, E.J., et al. 2008. Activation of PDGF-CC by tissue plasminogen activator impairs blood-brain barrier integrity during ischemic stroke. Nat. Med. 14:731-737.

21. Austyn, J.M., and Gordon, S. 1981. F4/80, a monoclonal antibody directed specifically against the mouse macrophage. Eur. J. Immunol. 11:805-815.

22. Jander, S., Kraemer, M., Schroeter, M., Witte, O.W., and Stoll, G. 1995. Lymphocytic infiltration and expression of intercellular adhesion molecule- 1 in photochemically induced ischemia of the rat cor- tex. J. Cereb. Blood Flow Metab. 15:42-51.

23. Hassan, N.F., Rifat, S., Campbell, D.E., McCawley, L.J., and Douglas, S.D. 1991. Isolation and flow cytometric characterization of newborn mouse brain-derived microglia maintained in vitro. J. Leukoc. Biol. 50:86-92.

24. Stirling, D.P., and Yong, V.W. 2008. Dynamics of the inflammatory response after murine spinal cord injury revealed by flow cytometry. J. Neurosci. Res. 86:1944-1958.

25. Gayle, R.B., 3rd. 1998. Inhibition of platelet function by recombinant soluble ecto-ADPase/CD39. J. Clin. Invest. 101:1851-1859.

26. Yu, L., et al. 2004. Selective inactivation or reconstitution of adenosine $\mathrm{A} 2 \mathrm{~A}$ receptors in bone marrow cells reveals their significant contribution to the development of ischemic brain injury. Nat. Med. 10:1081-1087.

27. Dwyer, K.M., et al. 2004. Thromboregulatory manifestations in human CD39 transgenic mice and the implications for thrombotic disease and transplantation. J. Clin. Invest. 113:1440-1446.

28. Freyer, D.R., et al. 1988. Modulation of surface CD11/CD18 glycoproteins (Mo1, LFA-1, p150,95) by human mononuclear phagocytes. Clin. Immunol. Immunopathol. 46:272-283.

29. Morabito, L., et al. 1998. Methotrexate and sulfasalazine promote adenosine release by a mechanism that requires ecto- 5 '-nucleotidase-mediated conversion of adenine nucleotides. J. Clin. Invest. 101:295-300.

30. Soto, F., Garcia-Guzman, M., and Stuhmer, W. 1997. Cloned ligand-gated channels activated by extracellular ATP (P2X receptors). J. Membr. Biol. 160:91-100.

31. Haines, W.R., Torres, G.E., Voigt, M.M., and Egan, T.M. 1999. Properties of the novel ATP-gated ionotropic receptor composed of the P2X(1) and P2X(5) isoforms. Mol. Pharmacol. 56:720-727.

32. Communi, D., Robaye, B., and Boeynaems, J.M. 1999. Pharmacological characterization of the human P2Y11 receptor. Br. J. Pharmacol. 128:1199-1206.

33. Tsuda, M., et al. 2003. P2X4 receptors induced in spinal microglia gate tactile allodynia after nerve injury. Nature. 424:778-783.

34. Inoue, K., Tsuda, M., and Koizumi, S. 2004. ATPand adenosine-mediated signaling in the central nervous system: chronic pain and microglia: involvement of the ATP receptor P2X4. J. Pharmacol. Sci. 94:112-114.

35. Murgia, M., Hanau, S., Pizzo, P., Rippa, M., and Di Virgilio, F. 1993. Oxidized ATP. An irreversible inhibitor of the macrophage purinergic $\mathrm{P} 2 \mathrm{Z}$ receptor. J. Biol. Chem. 268:8199-8203.

36. Michel, A.D., Kaur, R., Chessell, I.P., and Humphrey, P.P. 2000. Antagonist effects on human P2X(7) receptor-mediated cellular accumulation of YO-PRO-1. Br. J. Pharmacol. 130:513-520.

37. Arumugam, T.V., et al. 2004. Contributions of LFA-1 and Mac- 1 to brain injury and microvascular dysfunction induced by transient middle cerebral artery occlusion. Am. J. Physiol. Heart Circ. Physiol. 287: $\mathrm{H} 2555-\mathrm{H} 2560$.

38. Pulte, E.D., et al. 2007. CD39/NTPDase-1 activity and expression in normal leukocytes. Thromb. Res. 121:309-317.

39. Marcus, A.J., et al. 1982. Formation of leukotrienes and other hydroxy acids during platelet-neutrophil interactions in vitro. Biochem. Biophys. Res. Commun. 109:130-137.

40. Freedman, J.E., and Loscalzo, J. 2002. Plateletmonocyte aggregates: bridging thrombosis and inflammation. Circulation. 105:2130-2132.

41. Guckelberger, O., et al. 2004. Beneficial effects of
CD39/ecto-nucleoside triphosphate diphosphohydrolase- 1 in murine intestinal ischemia-reperfusion injury. Thromb. Haemost. 91:576-586.

42. Grenz, A., et al. 2007. Contribution of E-NTPDase1 (CD39) to renal protection from ischemia-reperfusion injury. FASEB J. 21:2863-2873.

43. Koziak, K., Sevigny, J., Robson, S.C., Siegel, J., and Kaczmarek, E. 1999. Analysis of CD39/ATP diphosphohydrolase (ATPDase) expression in endothelial cells, platelets, and leukocytes. Thromb. Haemost. 82:1538-1544.

44. Goepfert, C., et al. 2001. Disordered cellular migration and angiogenesis in cd39-null mice. Circulation. 104:3109-3115.

45. Hentzen, E.R., et al. 2000. Sequential binding of CD11a/CD18 and CD11b/CD18 defines neutrophil capture and stable adhesion to intercellular adhesion molecule-1. Blood. 95:911-920.

46. Lambrecht, G. 2000. Agonists and antagonists acting at P2X receptors: selectivity profiles and functional implications. Naunyn Schmiedebergs Arch. Pharmacol. 362:340-350.

47. Goepfert, C., et al. 2000. CD39 modulates endothelial cell activation and apoptosis. Mol. Med. 6:591-603.

48. Di Virgilio, F. 2007. Liaisons dangereuses: P2X(7) and the inflammasome. Trends Pharmacol. Sci. 28:465-472.

49. Soriano, S.G., et al. 1999. Mice deficient in Mac-1 (CD11b/CD18) are less susceptible to cerebral ischemia/reperfusion injury. Stroke. 30:134-139.

50. Hata, R., et al. 1998. A reproducible model of middle cerebral artery occlusion in mice: hemodynamic, biochemical, and magnetic resonance imaging. J. Cereb. Blood Flow Metab. 18:367-375.

51. Yegutkin, G.G., Samburski, S.S., and Jalkanen, S. 2003. Soluble purine-converting enzymes circulate in human blood and regulate extracellular ATP level via counteracting pyrophosphatase and phosphotransfer reactions. FASEB J. 17:1328-1330.

52. Thompson, L.F., et al. 1979. Ecto-5'-nucleotidase activity in $\mathrm{T}$ and $\mathrm{B}$ lymphocytes from normal subjects and patients with congenital X-linked agammaglobulinemia. J. Immunol. 123:2475-2478.

53. Day, S.M., et al. 2005. Macrovascular thrombosis is driven by tissue factor derived primarily from the blood vessel wall. Blood. 105:192-198.

54. Michelson, A., Barnard, M., Krueger, L., Valeri, C., and Furman, M. 2001. Circulating monocyte-platelet aggregates are a more sensitive marker of in vivo platelet activation than platelet surface P-selectin: studies in baboons, human coronary intervention, and human acute myocardial infarction. Circulation. 104:1533-1537.

55. The Jackson Laboratory. 2008. Tg(pPGKneobpA)3Ems, quantitative PCR, version 1.JAX Mice Protocol Index. http://jaxmice.jax.org/pub-cgi/protocols/protocols. sh?objtype=protocol\&protocol_id=1646.

56. Soliman, G.A., et al. 2007. A simple qPCR-based method to detect correct insertion of homologous targeting vectors in murine ES cells. Transgenic Res. 16:665-670.

57. Coppi, M.V., and Guidotti, G. 1997. Intracellular localization of Na,K-ATPase alpha2 subunit mutants. Arch. Biochem. Biophys. 346:312-321.

58. Cao, C., Lawrence, D.A., Strickland, D.K., and Zhang, L. 2005. A specific role of integrin Mac-1 in accelerated macrophage efflux to the lymphatics. Blood. 106:3234-3241.

59. Chopp, M., et al. 1994. Postischemic administration of an anti-Mac-1 antibody reduces ischemic cell damage after transient middle cerebral artery occlusion in rats. Stroke. 25:869-875; discussion 875-866. 